\title{
PIM1 kinase regulates cell death, tumor growth and chemotherapy response in triple-negative breast cancer
}

\author{
Fara Brasó-Maristany ${ }^{1}$, Simone Filosto ${ }^{1}$, Steven Catchpole ${ }^{1}$, Rebecca Marlow ${ }^{1}$, Jelmar \\ Quist $^{1,2}$, Erika Francesch-Domenech ${ }^{1}$, Darren A Plumb ${ }^{1}$, Leila Zakka ${ }^{1}$, Patrycja Gazinska ${ }^{1}$, \\ Gianmaria Liccardi ${ }^{3}$, Pascal Meier ${ }^{3}$, Albert Gris-Oliver ${ }^{4}$, Maggie Chon U Cheang ${ }^{3}$, Anna \\ Perdrix-Rosell ${ }^{1}$, Manar Shafat ${ }^{1}$, Elodie Noël ${ }^{1}$, Nirmesh Patel ${ }^{1}$, Kristen McEachern ${ }^{5}$, \\ Maurizio Scaltriti 6,7 , Pau Castel ${ }^{6}$, Farzana Noor ${ }^{1}$, Richard Buus ${ }^{3}$, Sumi Mathew ${ }^{1}$, Johnathan \\ Watkins ${ }^{1}$, Violeta Serra ${ }^{4}$, Pierfrancesco Marra ${ }^{1}$, Anita Grigoriadis ${ }^{1,2}$, and Andrew N Tutt ${ }^{1,3}$ \\ ${ }^{1}$ Breast Cancer Now Research Unit, Division of Cancer Studies, Faculty of Life Sciences and \\ Medicine, King's College London, Guy's Hospital, London, UK
}

${ }^{2}$ Cancer Bioinformatics, Division of Cancer Studies, Faculty of Life Sciences and Medicine, King's College London, London, UK

${ }^{3}$ Breast Cancer Now Toby Robins Research Centre, Institute of Cancer Research, London, UK ${ }^{4}$ Experimental Therapeutics Group, Vall d'Hebron Institute of Oncology, Barcelona, Spain ${ }^{5}$ Oncology iMed, AstraZeneca, Waltham, Massachusetts, USA

${ }^{6}$ Human Oncology and Pathogenesis Program (HOPP), Memorial Sloan Kettering Cancer Center, New York, New York, USA

${ }^{7}$ Department of Pathology, Memorial Sloan Kettering Cancer Center, New York, New York, USA

\begin{abstract}
Triple-negative breast cancers (TNBCs) have poor prognosis and lack targeted therapies. Here we identified increased copy number and expression of the PIM1 proto-oncogene in genomic data sets of patients with TNBC. TNBC cells, but not nonmalignant mammary epithelial cells, were
\end{abstract}

\footnotetext{
Correspondence should be addressed to A.N.T (andrew.tutt@icr.ac.uk).

Accession codes. Gene Expression Omnibus: raw data from the gene expression microarray for the Guy's Hospital TNBC-enriched data set are available under accession number GSE40267. ArrayExpress: SNP6.0 array experiments for the Guy's Hospital TNBCenriched data set are available under accession number E-MTAB-2626; breast cancer cell line gene expression data are available under the accession number E-TABM-157. European Genome-phenome Archive: the METABRIC Data Access Committee approved access to EGAD00010000164 and EGAD00010000162 data sets. The TCGA Data Access Committee approved access to TCGA BRCA data, which was downloaded as raw gene expression and Affymetrix SNP6.0 array data.

Author Contributions

A.N.T., A.G., F.B.-M., S.F. and P. Marra conceived and designed the research; F.B.-M. and S.F. performed the in vitro experiments with crucial help from S.C., E.N., S.M., M. Shafat, N.P. and A.P.-R.; J.W. and J.Q. performed the bioinformatics analyses; R.M., E.F.D., D.A.P. and L.Z. performed the cell line xenograft experiments; V.S. and A.G.-O. performed the PDX experiments; P.G., E.F.-D., L.Z. and F.N. performed IHC stainings; K.M. provided AZD1208 and expertise, and assisted in the design of in vitro and in vivo AZD1208 experiments; G.L. and P. Meier performed dynamic BH3 profiling analysis; R.B. performed the NanoString nCounter experiments; M.C.U.C. performed the statistical analysis of the gene expression analysis; P.C. and M. Scaltriti performed western blot analysis with the PIM1 NOV22-39-5 antibody, and F.B.-M. analyzed most of the results; F.B.-M. and A.N.T wrote the manuscript; A.N.T. coordinated this work; and all authors helped with data interpretation and manuscript editing.

Competing Financial Interests

The authors declare no competing financial interests
} 
dependent on PIM1 for proliferation and protection from apoptosis. PIM1 knockdown reduced expression of the anti-apoptotic factor BCL2, and dynamic BH3 profiling analysis revealed that PIM1 prevents mitochondrial-mediated apoptosis in TNBC cell lines. In TNBC tumors and their cellular models, PIM1 expression was associated with several transcriptional signatures involving the transcription factor MYC, and PIM1 depletion in TNBC cell lines decreased, in a MYCdependent manner, cell population growth and expression of the MYC target gene MCL1. Treatment with the pan-PIM kinase inhibitor AZD1208 impaired the growth of both cell line and patient-derived xenografts and sensitized them to standard-of-care chemotherapy This work identifies PIM1 as a malignant-cell-selective target in TNBC and the potential use of PIM1 inhibitors for sensitizing TNBC to chemotherapy-induced apoptotic cell death.

TNBC cells are defined by the lack of estrogen receptor (ER), progesterone receptor (PR) and human epidermal growth factor receptor 2 (HER2). No targeted therapy is currently available for TNBC, and the mainstay of therapy remains chemotherapy, to which many patients are highly resistant. Identification of novel drug targets, especially those that might affect chemotherapy resistance, is an important unmet medical need1. Chemotherapyresistant TNBCs are associated with very poor prognosis2,3 and often display coamplification and overexpression of the $M Y C$ oncogene4 (which encodes c-MYC) and its transcriptional target $M C L 1$ (ref. 5), which encodes an anti-apoptotic factor.

Previous studies identified a recurrent amplification in TNBC cells in the genomic region 6p21-p25 (refs. 6-8), which includes PIM1. PIM1, a member of the PIM kinase family, has been implicated in the control of cancer cell proliferation, migration and apoptosis9, particularly in prostate cancer and leukemia10,11. Pim1-null mice develop normally and are fertile12, indicating that PIM1 is not essential for physiologic tissue homeostasis and suggesting that side effects would be manageable if PIM1 was targeted specifically for therapy13. Indeed, a number of specific and potent PIM inhibitors have been developed13,14. Yet, to optimize drug positioning it is critical to understand the tumor types and specific molecular frameworks in which PIM1's function is essential.

This study was undertaken to test the hypothesis that PIM1 provides a proliferation advantage to TNBC cells and raises the threshold for cell death, such that TNBC cells are addicted to PIM1 for protection from spontaneous and chemotherapy-induced apoptosis. We identified cellular and molecular mechanisms that underpin TNBC's cellular addiction to PIM1, including a functional link between PIM1 and c-MYC and the effects of PIM1 on expression of the anti- apoptotic proteins MCL1 and BCL2, as well as on the expression of known regulators of malignant phenotypes in TNBC, such as the tyrosine phosphatase SHP2 (ref. 15) (encoded by PTPN11) and EPH receptor A2 (EPHA2)16,17. Accordingly, the panPIM kinase inhibitor AZD1208 (ref. 11) selectively sensitized PIM1-addicted TNBC cell models and patient-derived xenografts (PDXs) to standard-of-care chemotherapy. 


\section{Results}

\section{Copy number-dependent PIM1 gene expression in TNBC}

PIM1 is located on chromosome 6p21-p25, a recurrent amplicon in TNBC6-8. We investigated whether PIMI's copy number status and expression level are increased in TNBC by interrogating three independent published data sets: the Guy's Hospital TNBCenriched cohort18,19, the Cancer Genome Atlas (TCGA) Breast cohort20 and the METABRIC21 cohort. PIM1 mRNA levels were significantly higher in TNBCs than in nonTNBCs (Fig. 1a). PAM50 classification22 of these data sets demonstrated increased PIM1 expression levels in the basal-like molecular subtype (Supplementary Fig. 1a). PIM1 gene expression showed significant correlation with its copy number in TNBC cells in the Guy's Hospital and TCGA cohorts (Fig. 1b), and in basal-like tumors in the METABRIC and TCGA data sets (Supplementary Fig. 1b). A considerable amount of gene expression variability was observed across the data sets. Nevertheless, $75-85 \%$ of basal-like breast cancers showed PIM1 expression levels that are significantly higher than the top quartile for PIM1 expression levels in breast cancers of the non-TNBC HER2-enriched, luminal A and luminal B molecular subtypes (Supplementary Fig. 1c). Notably, such upregulation is underpinned by copy number gains, including amplifications, in a substantial proportion of TNBCs. The poor performance of PIM1-specific antibodies for use in immunohistochemistry applications precluded analysis of protein abundance in a large tumor series. However, both PIM1 mRNA (Fig. 1c) and PIM1 protein levels (Fig. 1d,e) were increased in cellular models of TNBC as compared to those in models of non-TNBC.

\section{Selective PIM1 kinase addiction in cellular models of malignant breast cancer}

To investigate PIM1's specific role in cells derived from malignant breast cancers, we used a TNBC-enriched cell line panel23,24 and three normal breast epithelial cell models. We assessed the effect of PIM1 knockdown on cell population growth by using a fluorescentdye-based cell viability assay and multiple siRNAs and shRNAs. The specificity of the siRNAs and shRNAs for PIM1 knockdown versus PIM2 and PIM3 knockdown was validated by standard qRT-PCR (Supplementary Fig. 2).

We used two distinct PIM1-specific shRNAs and a nontargeting (NT) control and found that four of five TNBC cell lines were sensitive to PIM1 knockdown, in contrast to luminal BT474 and nonmalignant HMEC cells (Fig. 2a). Comparable results were obtained using Hoechst-33342 staining to measure cell growth (Supplementary Fig. 3), demonstrating that the reduction in metabolic activity that was measured by the fluorescent-dye-based assay reflected a reduction in total cell number.

Next we tested a broader range of breast cancer and nonmalignant models by using transient transfection of siRNAs targeting PIM1 (Supplementary Fig. 4a). PIM1 silencing impaired the growth of six of seven TNBC cell lines; the exception was SUM159, a cell line that expresses low levels of PIM1. Two of four non-TNBC models showed a degree of PIM1 dependency under these conditions. Of note, none of the nonmalignant cell models were sensitive to PIM1 knockdown. There was a marked difference in response to PIM1 
knockdown between malignant and nonmalignant cells (Supplementary Fig. 4b), consistent with previous findings that Pim1-knockout mice have a normal phenotype12.

To exclude off-target effects, we performed rescue-of-function experiments by expressing a shRNA- and siRNA-resistant PIM1 construct that encodes a V5-tagged variant of PIM1 (hereafter referred to as V5-PIM1 ${ }^{\mathrm{R}}$ ) in SUM149 cells that were transduced with a shRNA targeting endogenous PIM1. PIM1 knockdown reduced cell population growth in $\beta$ galactosidase (LacZ)-expressing control cells but was ineffective in V5-PIM1 ${ }^{\mathrm{R}}$-expressing cells (Fig. 2b). Moreover, we generated cell lines that stably expressed a doxycyclineinducible shRNA- and siRNA-resistant PIM1 construct that encoded a hemaglutinin (HA)-

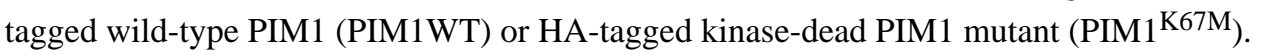
Expression of PIM1 ${ }^{\text {WT }}$ but not PIM1 ${ }^{\text {K67M }}$ rescued the effect of PIM1 knockdown in SUM149 cells (Supplementary Fig. 4c), indicating that the cell growth defect caused by PIM1 knockdown was specific to PIM1 and that the ability of PIM1 to promote cell growth depends on the activity of its kinase domain.

Next we investigated the effects of PIM1 silencing on TNBC clonogenic survival capacity. PIM1 knockdown reduced colony formation of TNBC cell lines, with the exception of the SUM159 cells, which express low levels of PIM1 (Fig. 2c). This result indicates a requirement of PIM1 for cell survival and/or proliferation across multiple cell division events. We did not observe effects of PIM2 or PIM3 silencing on cell population growth in the TNBC cell lines MDA-MB-231, SUM149 or SUM159 (Supplementary Fig. 5).

\section{PIM1 prevents activation of mitochondrial-mediated apoptosis in TNBC cells}

Time-course experiments in MDA-MB-231 cells showed that, following siRNA-mediated PIM1 knockdown, an increase in caspase- 3 and caspase- 7 activity (a marker for early apoptosis induction; hereafter referred to as caspase-3/7 activity) preceded the impairment of cell population growth (Fig. 3a,b). This result suggested that PIM1's effect on TNBC growth is at least in part due to protection from apoptosis. PIM1 knockdown, using multiple RNA interference (RNAi) methods, led to increased caspase 3/7 activity in only the TNBC cell lines in which we had documented a population growth defect (Supplementary Fig. 6a,b).

Next we examined the effect of PIM1 knockdown on the expression of the anti-apoptotic protein BCL2 in TNBC cells, as BCL2 is known to be under the control of PIM1 in other contexts25,26. PIM1 knockdown reduced the expression of BCL2 in MDA-MB-231 and SUM149 cells but did not affect BCL2 expression in the nonresponsive SUM159 and BT474 cell lines (Fig. 3c and Supplementary Fig. 7), implicating BCL2 regulation as a potential mechanism by which PIM1 protects TNBC cells from apoptosis. To determine whether PIM1's effect on caspase activation is mediated through BCL2, we silenced PIM1 in BCL2overexpressing MDA-MB-231 cells and found that BCL2 expression led to suppression of PIM1 knockdown-induced caspase 3/7 activation (Fig. 3d).

BCL2 inhibits apoptosis that arises from the mitochondrial apoptotic pathway27. We performed dynamic $\mathrm{BH} 3$ profiling (DBP), a recently developed methodology to measure changes in pro-apoptotic signaling at mitochondria28, to test the effect of PIM1 on the threshold at which increasing concentrations of a fragment of the pro-apoptotic protein 
BCL-2-interacting mediator of cell death (BCL2L11; also known as BIM) promotes apoptosis (Supplementary Fig. 8). At a BIM peptide concentration of $0.3 \mu \mathrm{M}$, PIM1 knockdown led to a reduction of this threshold in MDA-MB-231 and SUM149 cells but not in SUM159 cells. Ectopic expression of BCL2 in MDA-MB-231 cells fully rescued the effects of PIM1 knockdown on mitochondrial membrane permeabilization (Fig. 3e), suggesting that PIM1 prevents mitochondrial-mediated apoptosis in TNBC at least in part by modulation of BCL2.

Despite the ability of BCL2 overexpression to fully rescue the effect of PIM1 knockdown on enhancing caspase activation, BCL2 overexpression only partially rescued the cell growth defect caused by PIM1 knockdown (Fig. 3f). This result suggests that protection from the mitochondrial-apoptotic pathway is not the only mechanism by which PIM1 promotes cell growth.

\section{PIM1 acts through the MYC-activation pathway}

PIM1 has been found to synergize with the $M Y C$ oncogene to drive the progression of prostate cancer and leukemia10,29. Transcriptional activation by c-MYC relies on PIM1dependent phosphorylation of histone H3 at Ser10 in HEK293 cells and transformed rat fibroblasts30, and PIM1-dependent phosphorylation of c-MYC at Ser62 causes oncogenic transformation in prostate cancer10. Because $M Y C$ is known to be amplified and overexpressed in aggressive chemotherapy-resistant TNBC4, we tested the effects of PIM1 on histone H3 and c-MYC in TNBC cells and the relationship of these effects to the PIM1addiction status of the cells. PIM1 knockdown led to decreased levels of phosphorylated histone $\mathrm{H} 3$ at Ser10 and phosphorylated c- MYC at Ser62, as well as a reduction of total protein levels of c-MYC and its transcriptional target5 MCL1, in MDA-MB-231 and SUM149 cells (PIM1-knockdown sensitive) but not in SUM159 and BT474 cells (PIM1knockdown insensitive) (Fig. 4a and Supplementary Fig. 9a). However, SUM159 cells were sensitive to $M Y C$ knockdown (Supplementary Fig. 9b), indicating that the MYC activation pathway is likely to be controlled by other mechanisms 31,32 in this cell line.

MCL1 mRNA levels were also reduced after PIM1 knockdown (Fig. 4b), suggesting a mechanism in which PIM1 regulates MYC-driven MCL1 transcription. However, $M Y C$ mRNA levels were not affected after PIM1 knockdown, indicating that PIM1 regulates MYC at the protein level (Fig. 4b). To further study the involvement of MYC, we engineered TNBC cell lines in which c-myc expression is doxycycline inducible33. c-myc expression abolished the effects of PIM1 knockdown on cell population growth (Fig. 4c), indicating that PIM1 functions through c-MYC and its downstream effectors. Moreover, c-myc overexpression rescued the effects of PIM1 knockdown on MCL1 expression (Fig. 4c). Taken together, these data indicate that PIM1 has direct effects on c-MYC-driven transcription.

Next we investigated whether PIM1 and $M Y C$ gene expression correlate with each other in the Guy's Hospital18,19, TCGA Breast20 and METABRIC21 cohorts. Because we had found a moderate positive correlation (Supplementary Fig. 9c), we sought evidence of functional consequences of c-MYC-driven transcription. Thirty-four c-MYC-dependent transcriptional signatures have been reported (Supplementary Table 1). Among the first of 
these was that by Chandriani et al.34, which was obtained from primary human fibroblasts overexpressing c-MYC and found to be associated with basal-like breast cancer34,35. This signature was upregulated in TNBC tumors and cellular models (Supplementary Fig. 9d). Next we determined the activation score of all 34 gene signatures across the three cohorts and the breast cancer cell line data set23, using a weighted average mean 36 . The activation scores were then correlated to PIM1 expression. We found a modest but significant correlation for eight of the MYC-dependent transcriptional signatures in all four data sets (Supplementary Table 1). The correlation between Chandriani's c-MYC activation signature and PIM1 expression in the four data sets is depicted in Figure 4d.

We then asked whether PIM1 has a direct role in driving expression of the genes in the Chandriani c-MYC gene signature, the activation score of which was associated with PIM1 upregulation in basal-like breast cancer (Fig. 4d). We performed NanoString nCounter PanCancer Pathways code set gene expression analysis following PIM1 knockdown using multiple RNAi methods. The reduction in PIM1 mRNA levels after PIM1 silencing was confirmed for each cell line (Supplementary Fig. 9e). Of the 24 genes in the Chandriani signature whose expression is dependent on c-MYC and could be interrogated in the code set, 11 were downregulated by PIM1 knockdown in the PIM1-knockdown-sensitive cell lines (Supplementary Fig. 9f and Supplementary Table 2). In the PIM1-knockdowninsensitive SUM159 cell line, in which PIM1 knockdown was not observed to affect the levels of c-MYC protein, c-MYC phosphorylation at Ser62 or histone H3 phosphorylation at Ser10 (Fig. 4a), only five of the genes were downregulated. We suggest that PIM1 regulation of MYC- dependent transcription in TNBC will only partially overlap with that seen in the fibroblast model system, which may explain why we found that only a subset of the c-MYC target genes were downregulated after PIM1 knockdown.

Next we investigated the effect of PIM1 knockdown on a wider set of transcriptional responses in the PIM1-knockdown-sensitive MDA-MB-231 and SUM149 cell lines, using the entire nCounter PanCancer Pathways gene expression code set (Fig. 4e). We sought significant changes in gene expression $\left(0.5<\log _{2}\right.$-fold changes $\left.<-0.5 ; P \leq 0.05\right)$ and found significant effects on genes of the JAK-STAT signaling pathway, as well as on genes involved in apoptosis and the cell cycle, consistent with known functions of PIM1 in other systems9 (Fig. 4e and Supplementary Table 3). PIM1 knockdown led to increased expression of cyclin-dependent kinase inhibitor 1B ( $C D K N 1 B$; which encodes the cell cycle inhibitor p27), which was previously shown to be regulated by PIM1 (ref. 37). Moreover, PIM1 knockdown led to decreased expression of PTPN11 (whose product, also known as SHP2, is known to be important for maintenance of tumor-initiating cells and clonogenic survival in TNBC)15 and of EPHA2 (which encodes a regulatory kinase for SHP2)38. These results were validated at the protein level (Fig. 4f) and suggest additional mechanisms by which PIM1 regulates malignant cell phenotypes in TNBC.

\section{AZD1208 inhibits in vitro and in vivo growth of TNBC models and enhances chemosensitivity}

AZD1208 is a highly selective inhibitor of all three PIM kinases11. To examine the potential of using PIM inhibitors to target PIM1-dependent TNBCs, we treated TNBC cell lines and 
the normal breast epithelial cell line MTSV1.7 with AZD1208 in colony-formation assays. Consistent with the RNAi results, MDA-MB-231 and SUM149 cells were sensitive to PIM kinase inhibition, in contrast to SUM159 and MTSV1.7 cells (Fig. 5a,b). AZD1208 treatment also substantially reduced anchorage-independent growth of TNBC cells (Supplementary Fig. 10). Moreover, consistent with the effects of PIM1-specific RNAi treatment, AZD1208 treatment reduced MCL1 and BCL2 protein levels and reduced the levels of phosphorylated histone H3 at Ser10 and phosphorylated c-MYC at Ser62 (Fig. 5c).

Next we treated mice bearing MDA-MB-231 and SUM149 cell line xenografts with AZD1208. This treatment substantially impaired tumor growth (Fig. 5d,e) and induced an increase in the percentage of p27-positive cells (Supplementary Fig. 11), consistent with the gene expression analysis and with the known role of PIM1 in cell cycle progression through p27 regulation37.

Consistent with the effects of PIM1 knockdown and the failure of kinase-dead PIM1 to rescue the knockdown-dependent phenotypes, AZD1208 treatment of MDA-MB-231 and SUM149 cells (but not of SUM159 cells) led to mitochondrial membrane permeabilization at a BIM peptide dose of $0.3 \mu \mathrm{M}$, as assessed by DBP (Fig. $5 \mathrm{f}$ and Supplementary Fig. 12). Notably, BCL2 overexpression rescued this effect of PIM kinase inhibition.

Induction of apoptosis is a major contributor to the therapeutic effect of chemotherapy, which remains the mainstay of TNBC treatment despite limited efficacy in metastatic disease39. Treatment of TNBC cells with the standard advanced TNBC chemotherapy agents eribulin and paclitaxel increased caspase $3 / 7$ activity, an effect which was further increased by AZD1208 treatment in the PIM1- knockdown-sensitive MDA-MB-231 cell line but not in the PIM1- knockdown-insensitive SUM159 cell line (Fig. 5g). Moreover, BCL2 overexpression prevented mitochondrial-membrane permeabilization that was induced by combined treatment with eribulin and AZD1208 (Supplementary Fig. 13).

We next investigated the therapeutic potential of combining PIM1 inhibition with the use of chemotherapeutic agents in TNBC cell lines. AZD1208 treatment sensitized MDA-MB-231 and SUM149 cells, but not SUM159 or MTSV1.7 cells, to paclitaxel (Fig. 6a). AZD1208 treatment also sensitized MDA-MB-231 and SUM149 cells, but not SUM159 cells, to eribulin (Fig. 6b). The effect of AZD1208 on the sensitivity of MTSV1.7 cells to eribulin treatment could not be tested because of the exquisite sensitivity of these cells to eribulin (data not shown). The efficacy of combination therapy in reducing long-term colony formation in PIM1-sensitive models is consistent with the observation that addition of AZD1208 to standard-of-care chemotherapy increased caspase 3/7 activity (Fig. 5g). Taken together, these findings suggest that the effect of combination therapy on colony formation is substantially mediated through induction of apoptosis.

We then tested the ability of PIM1 inhibition to sensitize TNBC xenografts and PDX models to chemotherapeutic treatment. We treated two TNBC cell line xenograft models (Fig. 6c,d) and two TNBC PDX models (Fig. 6e,f) (which display high levels of PIM1; Supplementary Fig. 14a) with eribulin and AZD1208. Eribulin treatment alone led to stabilization of disease, whereas the combination of eribulin and AZD1208 led to objective treatment 
responses in the xenografts (Fig. 6c-f and Supplementary Fig. 14b-g) and marked reductions in viable tumor cell density and an increase in fibrosis at the tumor implantation site (Fig. $6 \mathrm{~g}-\mathrm{n}$ ), demonstrating the potential efficacy of combining PIM kinase inhibition with eribulin treatment. The PIM kinase inhibitor, despite its known activity against mouse PIM1 (ref. 40), did not have effects on animal weight (Supplementary Fig. 14h-k), distress or lethality (data not shown), when either given alone or in combination with chemotherapy, supporting the notion that PIM inhibition could be well tolerated in patients.

\section{Discussion}

Our data identify PIM1 kinase as a potential therapeutic target in TNBC. We demonstrate elevated expression of PIM1 and frequent copy number aberrations (CNAs) of gene in a high proportion of basal-like TNBC. By using complementary approaches of RNAi knockdown and a small-molecule kinase inhibitor, we demonstrated that PIM1 kinase activity is required for the survival of TNBC cells both in vitro and in vivo, especially when combined with the apoptotic stimulus of chemotherapy.

TNBC includes a number of biologically distinct entities 17. Whereas genome-profiling studies of TNBC samples indicate a substantial diversity of driver mutations, most of which occur at low frequency, some mutations and gene CNAs are recurrent. For example, oncogenic $M Y C$ is frequently amplified or overexpressed, and it drives oncogenic pathway activation and tumorigenicity 17,35 . c-MYC is also associated with poor prognosis 41 and chemotherapy resistance in TNBC4, but this protein is notoriously hard to target 42 . Our results demonstrate not only that most TNBC cell lines are addicted to PIM1 for survival and proliferation but also that PIM1 functions in part through its effects on c-MYC. Specifically, c-MYC overexpression abrogated the PIM1-knockdown cell growth phenotype; c-MYCdependent transcriptional signatures were associated with PIM1 expression in TNBCs; and PIM1 knockdown downregulated transcription of a subset of c-MYC target genes (including $M C L 1$ ) and were associated with effects on phosphorylation of histone $\mathrm{H} 3$ at Ser10 and cMYC at Ser62, which are known to be required for c-MYC-driven transcriptional activation and oncogenic transformation10,30. Taken together, our data support PIM1 as a potential target in basal-like TNBCs, including in the c-MYC-driven group. Our data are also supported by an independent study by Horiuchi et al.43, which demonstrates that c-MYC overexpression generates a requirement of PIM1 function for cell growth in mammary epithelial cells and further indicates that PIM1 function is needed to support c-MYCdependent malignancy in TNBC cells.

$M Y C$ and $M C L 1$ are co-amplified in residual TNBC after neoadjuvant chemotherapy, leading to the proposal that $M C L 1$ amplification can lead to its overexpression and circumvent oncogene- and chemotherapy-induced apoptosis4. Moreover, most basal-like TNBCs show a high level of dependence on MCL1 (ref. 44). We found that MCL1 expression is increased after c-MYC overexpression in TNBC, consistent with work showing that c-MYC transcriptionally controls $M C L 1$ expression in gastric cancer cells5 and that PIM1 regulates c- MYC transcriptional activity, including MCL1 expression levels. PIM1 phosphorylates the BCL2 partner protein BAD in leukemia cells11 and kidney COS-7 cells25. Increased PIM1 expression in TNBC may overcome oncogene-induced and 
chemotherapy-induced apoptosis by increasing the threshold for mitochondrial-mediated apoptosis, which we suggest is due to the effects of PIM1 on BCL2 family members.

Similar to c-MYC, BCL2 and MCL1 are hard-to-target proteins27. Inhibitors of BCL2 family members have frequently shown off-target effects, triggering cell death independently of on-target mitochondrial-mediated apoptosis 27. Here we show that the kinase activity of PIM1 is specifically required for cell population growth and protection from apoptosis. Moreover, the pan-PIM kinase inhibitor AZD1208 could not only impair the clonogenicity of TNBC cells and the tumor growth of TNBC xenografts, but it also increased proapoptotic effects, and in vitro cellular and in vivo TNBC model sensitivity, to approved chemotherapeutic agents that are used in early and metastatic TNBC. Because specific and potent PIM1 inhibitors are in late stages of development14, PIM1 may be a more feasible drug target than BCL2 family members for overcoming resistance to chemotherapy. The current development focus for PIM inhibitors is in hematological malignancy45, but our data suggest that these agents can additionally be repurposed to address the major unmet clinical need in chemotherapy-resistant TNBC.

Besides the cooperation between PIM1 and c-MYC, multiple mechanisms could contribute to the effects of PIM1 on TNBC cell survival and proliferation. Indeed, gene expression analysis revealed PIM1- mediated regulation of the JAK-STAT pathway and of genes involved in apoptosis and the cell cycle, consistent with previously described PIM1 functions9. Specifically, we found that PIM1 knockdown upregulated expression of $C D K N 1 B$ (which encodes p27) at both the mRNA and protein level, suggesting a mechanism by which PIM1 promotes cell cycle progression in TNBC. Our findings are consistent with those of Horiuchi et al.43, as well as with work showing that PIM1 downregulates $C D K N 1 B$ at the transcriptional and post-transcriptional levels in other contexts37. In addition, PIM1 knockdown decreased expression of PTPN11 and EPHA2, genes of substantial relevance to breast cancer 15,16 that were not previously connected to PIM1. PTPN11 encodes the phosphatase SHP2, which is involved in breast cancer progression and the maintenance of tumor-initiating cells15. EPHA2 can regulate SHP2 function 38 and, together with PIM1, belongs to a cluster of kinases associated with poor prognosis in ER-negative breast cancer16,17.

We found that PIM1 expression was essential for cell growth and apoptosis protection in most of the malignant breast cell line models, including some of non-TNBC origin, but was expendable in nonmalignant cell models, including mammary epithelial cells. Indeed, although upregulation of PIM1 expression, particularly as driven by copy number aberration, is associated specifically with basal-like breast cancers, which form a large subset of TNBC, PIM kinase function may be relevant to a broader range of high-grade breast cancer, including ER-positive forms of the disease, as recently proposed by Malinen et al.46. Notably, Pim1-null mice develop normally and are fertile12, indicating that PIM1 may not be essential for physiologic tissue homeostasis, consistent with early evidence of good tolerability of PIM1 inhibitors in early-phase clinical trials47,48.

Our study provides insights into the mechanisms by which PIM1 drives malignant phenotypes in TNBC and provides a rationale for clinical trials to investigate PIM1 
inhibition combined with chemotherapy as a therapeutic strategy in basal-like TNBC cancers with high levels of PIM1 gene expression. Moreover, PIM1 inhibition may provide a much needed means of targeting tumors that amplify and express $M Y C$ and the co-amplified gene $M C L 1$ (ref. 5), which is also a transcriptional target of c-MYC, as well as other antiapoptotic BCL2 family members that underpin resistance to breast cancer chemotherapy.

\section{Online Methods}

\section{Cell lines}

The breast cancer cell lines BT20, BT474, CAL51 HCC1143, HCC1428, HCC1954, HCC38, MCF7, MDA-MB-231, MDA-MB-436 and SKBR3, as well as nonmalignant MCF10A cells, were from the American Type Culture Collection (ATCC, Teddington, UK). The breast cancer cell lines SUM149 and SUM159 were from Asterand (Royston, UK). The nonmalignant HMEC and MTSV-1.7 cells were from Invitrogen (Life Technologies, Paisley, UK). HEK293T cells were also from ATCC. MDA-MB-231 cells expressing pTIPZ-BCL2 or an RFP control were kindly provided by P. Meier's lab (The Institute of Cancer Research, London, UK). All cell lines were maintained as recommended by the suppliers. The cell lines were authenticated by short- tandem-repeat (STR) analysis and matched to the German Collection of Microorganisms and Cell Cultures (DSMZ) database, and they were used for no more than 25 passages after STR typing. Mycoplasma tests were routinely performed using MycoAlert Mycoplasma Detection Kit (Lonza, Basel, Switzerland). Although MCF7 and BT20 are included in the database of commonly misidentified cell lines, they were authenticated by STR, and we included them in our work as part of a comprehensive validation. Nevertheless, we focused on other cell lines for the main part of this study.

\section{Gene expression and DNA copy number analysis}

Gene expression data (PIM1, MYC and genes from 34 MYC transcriptional signatures34,49-60) were obtained from the Guy's Hospital TNBC-enriched cohort18,19 (177 primary breast tumors), the TCGA data set20 (526 primary breast tumors), the METABRIC data set21 (1,552 primary breast tumors) and a publicly available cell line data set23 (51 cell lines). Breast cancer specimens were classified as TNBC or non-TNBC based on immunohistochemistry (IHC), as per the guidelines of the American Society of Clinical Oncology (ASCO) and the College of American Pathologists (CAP)61, or into PAM50 molecular subtypes22. To identify subtypes based on the molecular PAM50 classification, a nearest-centroid classification was performed, as described previously 22 , for the METABRIC and TCGA cohorts. For the Guy's Hospital TNBC-enriched cohort, we adapted the PAM50 classification by performing random sampling19. Assignment of tumors to the molecular subtypes was based on their highest Spearman's rank correlation. All statistical analyses were performed in the R environment, using several CRAN packages (http://cran.rproject.org/). Absolute DNA copy number was obtained by using Affymetrix SNP6.0 genome profiles and human genome build hg18 mapping for 196 cancers in our Guy's Hospital cohort and for 940 primary breast carcinomas from the TCGA data set. Raw copy number information was processed for 1,501 breast cancers from the METABRIC data set. To account for normal tissue contamination and ploidy, allele-specific copy number profiling was performed with allele-specific copy number analysis of tumors (ASCAT)62. SNP 6.0 
copy number data were also extracted from our internal breast cancer cell line data set36 and analyzed using Tumor Aberration Prediction Suite (TAPS)63.

\section{Western blot analysis}

Cells were lysed directly in $1 \times$ Laemmli buffer $(50 \mathrm{mmol} / \mathrm{L}$ Tris-HCl pH 6.8, $2 \%$ SDS, $10 \%$ glycerol, $2.5 \% \beta$-mercaptoethanol and $0.1 \%$ bromophenol blue). Alternatively, cells were lysed by scraping them into a pH 7.4 lysis buffer containing 1\% NP-40 (Sigma-Aldrich, Gillingham, UK), $50 \mathrm{mmol} / \mathrm{L}$ Tris, $10 \%$ glycerol, $0.02 \% \mathrm{NaN}_{3}, 150 \mathrm{mmol} / \mathrm{L} \mathrm{NaCl}$, and a cocktail of phosphatase and protease inhibitors (Sigma-Aldrich, Gillingham, UK). Cell lysates were passed five times through a 30-gauge needle before centrifugation and further processing of the samples. Snap-frozen tumor tissues were suspended in a $\mathrm{pH} 7.4$ lysis buffer containing $50 \mathrm{mmol} / \mathrm{L}$ Tris base, $150 \mathrm{mmol} / \mathrm{L} \mathrm{NaCl}, 2 \%$ TritonX-100, 1\% SDS, 10 $\mathrm{mmol} / \mathrm{L}$ EDTA, and a cocktail of phosphatase and protease inhibitors (Sigma-Aldrich, Gillingham, UK). Tissue destruction was done with the bullet blender homogenizer (Next Advance, New York, USA). 20-100 $\mu \mathrm{g}$ of proteins were separated in reducing conditions (2.5\% $\beta$-mercaptoethanol) by SDS-PAGE (SDS-polyacrylamide gel electrophoresis) and transferred to nitrocellulose membranes (Bio-Rad, Hemel Hempstead, UK) for further processing, following standard western blotting procedures.

Primary antibodies used in this study were: anti-PIM1 rabbit monoclonal antibody (ab75776) (1/1,000), anti-c-MYC rabbit monoclonal antibody (ab32072) (1/2,000) and antiphospho-c-MYC(Ser62) rabbit polyclonal antibody (ab51156) (1/500) from Abcam (Cambridge, UK); anti-PIM1 rabbit antibody (PIM-1: NOV22-39-5) $(1 / 1,000)$ from Novartis (Basel, Switzerland); anti-PIM2 (D1D2) (\#4730) (1/1,000), anti-PIM3 (D17C9) (\#4165) $(1 / 1,000)$, anti-MCL1 (\#4572) (1/1,000), anti-BCL2 (50E3) (\#2870) (1/1,000), anti-histoneH3 (D1H2) XP (\#4499) (1/2,000), anti-phospho-histone-H3(Ser10) (D2C8) (\#3377) $(1 / 2,000)$ and anti-GAPDH (14C10) (\#2118) $(1 / 10,000)$ rabbit antibodies from Cell Signaling Technologies (Massachusetts, USA); anti- $\beta$-actin mouse antibody (A5441) $(1 / 30,000)$ from Sigma-Aldrich (Gillingham, UK); anti-V5 tag rabbit antibody from Life Technologies (Paisley, UK) (1/1,000); and anti-HA tag rabbit antibody (GTX115044) $(1 / 1,000)$ from GeneTex (Irvine, USA). The secondary peroxidase-conjugated anti-mouse $(1 / 50,000)$ and anti-rabbit antibodies $(1 / 50,000)$, as well as the enhanced chemiluminescence (ECL) substrate, were obtained from GE Healthcare (Amersham, UK). ImageJ was used to quantify western blotting results by densitometry. Uncropped western blots are shown in Supplementary Figs. 15 and 16

\section{Short hairpin RNA (shRNA)- and small interfering RNA (siRNA)-mediated gene knockdown}

Two pLKO.1-puro lentiviral MISSION shRNA constructs targeting PIM1 (TRCN0000010115 (\#1) and TRCN0000010118 (\#2)) and a nontargeting (NT) control shRNA (TRC1/1.5) were from Sigma-Aldrich (Gillingham, UK). The shRNA\#1 sequence targeting PIM1 and the NT shRNA were also subcloned into the inducible Tet-pLKO-puro lentiviral vector (Addgene, Massachusetts, USA). Lentiviral particles were produced in HEK293T cells and titrated onto breast cancer cells in medium containing puromycin (1.5 $\mu \mathrm{g} / \mathrm{ml}$ ) in order to achieve optimal knockdown of the target protein with minimal viral load. 
Transduced cells were treated with doxycycline $(0.5 \mu \mathrm{g} / \mathrm{ml})$ to achieve knockdown, where appropriate.

siGENOME siRNA targeting PIM1 (D-003923-01) or MYC (L-003282-02- 0005), and a nontargeting siRNA pool\#2 (D-001206-14) control, were purchased from Dharmacon (Thermo Scientific, Hemel Hempstead, UK). Silencer Select siRNA targeting PIM2 (s21751) was purchased from Ambion (Thermo Fisher Scientific, Massachusetts, USA). FlexiTube PIM3 siRNA\#1 (S100684915), PIM3 siRNA\#5 (S103084543) and a nontargeting siRNA (S103650325) were purchased from Qiagen Ltd. (Manchester, UK). Cells were transfected with $20 \mathrm{nmol} / \mathrm{L}$ siRNA using Lipofectamine RNAiMax transfection reagent (Invitrogen, Life Technologies, Paisley, UK), following the manufacturer's instructions.

\section{cDNA expression constructs}

The complete PIM1 cDNA sequence (NCBI reference sequence NM_001243186.1) subcloned into the pDONR221 entry vector was obtained from Geneart (Life Technologies, Paisley, UK). This cDNA sequence incorporated silent mutations in the third nucleotide of the codons of the sequence targeted by PIM1 shRNA\#1 and PIM1 siRNA, and the stop codon (TAG) was replaced with a tyrosine-encoding codon (TAC) to generate a rescue construct that is insensitive to the PIM1-specific shRNA. PIM1 cDNA from the entry vector was Gateway-recombined into the pLenti6.2/C-Lumio/V5-DEST vector by using the Virapower C-Lumio Lentiviral Expression kit (Life Technologies, Paisley, UK). A kinasedead PIM1 (PIM1 ${ }^{\text {K67M }}$ ) construct was generated by introducing a site- specific mutation into the complete PIM1 cDNA sequence (pDONR221 entry vector), mutating the codon encoding Lys67 to a codon encoding methionine, using the PCR-based site-directed mutagenesis kit (Stratagene, CA, USA). Primers that were used to introduce the mutation were designed following Stratagene guidelines. A $M Y C$ overexpression vector was obtained from Addgene (Massachusetts, USA)33. The PIMI ${ }^{\mathrm{WT}}, P I M I^{\mathrm{K} 67 \mathrm{M}}$ and $m y c$ sequences were Gateway-recombined into the pInducer20 vector, which has a sequence encoding an HA tag64. All of the constructs were sequenced by SourceBioscience (Cambridge, UK). Lentiviral particles were produced in HEK293T cells.

\section{Quantitative RT-PCR}

RNA was extracted from cells by using the QIAZOL reagent (Qiagen Ltd., Manchester, UK), following manufacturer's instructions. cDNA was prepared from $2 \mu \mathrm{g}$ total RNA in the presence of random hexamers and using an Applied Biosystems kit, according to manufacturer's instructions. PCR was performed using a SYBR-Green-based detection system and a 7900HT Fast RT-PCR thermocycler (Applied Biosystems, Massachusetts, USA) at the Guy's Hospital genomics laboratory. We used the following primers: $5^{\prime}$ CGAGCATGACGAAGAGATCAT-3' and $5^{\prime}$-TCGA AGGTTGGCCTATCTGA-3' for PIM1; 5-GAGTCAACGGATTTGGTCGT- ${ }^{\prime}$ and $5^{\prime}$-TTGATTTTGGAGGGATCTCG-3' for $G A P D H$; and 5-GGGCAGG ATTGTGACTCTCATT-3' and 5GATGCAGCTTTCTTGGTTTATGG-3' for $M C L 1$. Primers specific to $M Y C$ (QT00035406), PIM2 (QT00086884) and PIM3 (QT00092197) were obtained from Qiagen Ltd. (Manchester, UK). Each set of primers was validated following standard PCR procedures, which showed the generation of one product at only the expected molecular 
weight. GAPDH was used as a housekeeping target, and the data were normalized using a standard comparative $C_{\mathrm{t}}$ method.

\section{Cell population growth assay}

Cells were plated in triplicate at 4,000 cells/well in 96-well plates. Cell population viability was determined over time using Cell Titer-Blue (Promega, Southampton, UK), following the manufacturer's instructions, or by labeling DNA with Hoechst 33342 (Invitrogen, Life Technologies, Paisley, UK). Fluorescence was read using the FLUOstar Omega plate reader (BMG LabTech, Aylesbury, UK).

\section{Caspase $3 / 7$ activation assay}

Cells were plated in triplicate at 4,000 cells/well in 96-well plates. Caspase activation was determined using the Caspase-Glo3/7 Assay (Promega, Southampton, UK), following the manufacturer's instructions.

\section{Two-dimensional (2D) colony-formation assay}

Cells were plated in triplicate at a low density in 6-well plates. After 15-21 d, the cells were fixed with cold methanol and stained with $0.5 \%$ crystal violet solution. Colonies were counted with an e-count pen (Heathrow Scientific, Nottingham, UK)65. For drug treatments, paclitaxel and eribulin were provided as remnants by the Guy's Hospital Oncology Pharmacy. AZD1208 was provided by AstraZeneca (Wilmington, Delaware, USA). The culture medium was replaced every other day. For drug-combination treatments, AZD1208 or DMSO control was given $24 \mathrm{~h}$ before chemotherapeutic drug treatment. Cells were then treated with combinations of chemotherapeutic drugs and AZD1208 or DMSO control for $24 \mathrm{~h}$. Medium was then replaced, and AZD1208 or DMSO control was given every other day until the end of the experiment.

\section{Three-dimensional (3D) anchorage-independent growth assay in soft agar}

A total of 1,000 singly suspended MDA-MB-231 cells were mixed in culture medium $\left(37^{\circ} \mathrm{C}\right)$ containing $0.3 \%$ low-melting-point agar (Fermentas, Vilnius, Lithuania) and then seeded in 12-well plates on top of a layer of $0.6 \%$ agar (Life Technologies, Paisley, UK). AZD1208 (AstraZeneca) was administered in a top (feeding) culture medium, which was replaced every $4 \mathrm{~d}$. After 4 weeks in culture, the colonies (composed of at least $\sim 20$ cells) were counted by optical microscopy at $\times 200$ magnification.

\section{Dynamic BH3 profiling (DBP)}

$15 \mu \mathrm{l}$ of BIM BH3 peptide at four different concentrations $(0.2,0.6,2.0$, and $6.0 \mu \mathrm{M})$ was prepared in DTEB (300 mM trehalose, $10 \mathrm{mM}$ HEPES-KOH (pH 7.7), $80 \mathrm{mM} \mathrm{KCl,} 1 \mathrm{mM}$ EGTA, $1 \mathrm{mM}$ EDTA, $0.1 \% \mathrm{BSA}$ and $5 \mathrm{mM}$ succinate). $15 \mu \mathrm{l}$ of each peptide concentration was added to wells in triplicate using a black 384-well plate (NUNC). The plate was left at room temperature (RT) during the preparation of the single-cell suspension. Cells, which were previously plated at 150,000 cells/well in 6-well plates, were trypsinized and collected $72 \mathrm{~h}$ after siRNA (NT or PIM1) transfection, or following AZD1208 or DMSO treatment. Single-cell suspensions were pelleted at $500 \mathrm{~g}$, resuspended and washed in DTEB before 
being resuspended in DTEB at a density of $2.67 \times 10^{6}$ ( $4 \times$ density). One volume of the cell suspension was added to one volume of the $4 \times$ dye solution previously prepared ( $4 \mu \mathrm{M} \mathrm{JC}-1$, $40 \mu \mathrm{g} / \mathrm{ml}$ oligomycin, $0.02 \%$ digitonin, $20 \mathrm{mM} \beta$-mercaptoethanol in DTEB). The $2 \times$ cell/dye solution was left at RT for $10 \mathrm{~min}$ to allow permeabilization and dye equilibration. $15 \mu \mathrm{l}$ of the $2 \times$ cell/dye mix was then added to each treatment well of the plate. This led to a final concentration of the BIM peptide in each well of $0.1,0.3,1.0$ or $3.0 \mu \mathrm{M}$. The plate was then placed into the Infinite M200 pro TECAN plate reader. The plate was shaken for $15 \mathrm{~s}$, and individual wells were read at $590 \mathrm{~nm}$ every 5 min over a 3 -h time course at $30^{\circ} \mathrm{C}$. The obtained values were plotted as ' $\triangle$ priming'. The difference in priming was determined by calculating the area under the DMSO control and the FCCP control minus the area under the individual peptide concentrations and the FCCP. These differences where then plotted as $\Delta \Psi_{\mathrm{n}}$ at $\mathrm{RFU}_{590 \mathrm{~nm}}$.

\section{Nanostring nCounter PanCancer Pathways gene expression analysis}

RNA was extracted from cells using the QIAZOL reagent (Qiagen Ltd., Manchester, UK), following the manufacturer's instructions. Samples were quantified using Nanodrop (Thermo Scientific, Hemel Hempstead, UK) and Qubit Fluorimetric Quantitation (Thermo Scientific, Hemel Hempstead, UK). The NanoString nCounter System (NanoString Technologies, Seattle, WA, USA) was used to measure the gene expression profiles of 12 samples. 100 ng total RNA was assessed using the nCounter PanCancer Pathways Panel, which targets 730 genes representing all of the major cancer pathways, including key driver genes. Expression data were normalized using the nSolver analysis module and custom scripts in R 2.13.1. Background correction was done by subtracting the geometric mean of the eight negative-control probes. Expression values were normalized with the most stable 31 housekeeping genes, which were selected based on the geNorm algorithm. Expression values were then $\log _{2}$-transformed and standardized within each sample. All 730 genes in the present study were detected. Significant differential genes were identified by two-class comparison (control versus experimental condition); a volcano plot was used to display the fold change and $P$ value (using the software packages ggplot and calibrate in the R enviroment).

\section{Mouse studies}

All animal experiments with cell line xenografts were approved by the King's College London Institutional Committees on Animal Welfare, and in compliance with the United Kingdom Home Office Animals Scientific Procedures Act, 1986. Female CD-1 Nu/Nu mice were obtained from Charles River UK Ltd. Procedures were carried out on 28- to 35-d-old mice. One million MDA-MB-231 or SUM149 cells mixed 1:1 in PBS:Matrigel were injected into the inguinal fat pad of mice using standard procedures. After the tumors reached $4 \mathrm{~mm}$ in diameter, as assessed by palpation and caliper measurement, mice were randomized into groups (treatments with vehicle, AZD1208, eribulin, or combinations of eribulin and AZD1208). Mice were then dosed daily by oral gavage at $30 \mathrm{mg}$ per kg body weight (mg/kg) (MDA-MB-231 xenografts) or $15 \mathrm{mg} / \mathrm{kg}$ (SUM149 xenografts) of AZD1208 or vehicle control $(0.1 \%$ Tween-80, $0.5 \%$ methylcellulose). Eribulin was administered by intravenous tail-vein injection at $0.1 \mathrm{mg} / \mathrm{kg}$. Saline solution $(0.9 \%)$ was used as the vehicle control for eribulin treatment. Tumor growth was monitored over time. After vehicle-treated 
tumors reached $10 \mathrm{~mm}$ in diameter, the experiment was terminated, and tumors were excised and processed for western blotting analysis (snap-frozen) or fixation in formalin for histological examination (FFPE).

Patient-derived xenotransplants (PDX) studies were performed at the Experimental Therapeutics Group (Vall d'Hebron Institute of Oncology, Barcelona, Spain) and were approved by the Ethical Committee on Animal's Healthcare and under the Patient's Informed Consent of the Vall d'Hebron University Hospital. Foxn $1^{-/}$nude mice were obtained from Janvier Labs (Le Genest-Saint-Isle, France). Procedures were carried out in 42- to 56-d-old female mice. For each PDX model, one 2-mm tumor piece was implanted into both inguinal fat pads of mice following standard procedures. When tumors reached 6 $\mathrm{mm}$ in diameter, as assessed by palpation and caliper measurement, mice were randomized into groups (treatment with vehicle, AZD1208, eribulin, or combinations of eribulin and AZD1208). Animals were then dosed daily by oral gavage at $30 \mathrm{mg} / \mathrm{kg}$ AZD1208 or vehicle $(0.1 \%$ Tween $80,0.5 \%$ methyl cellulose). Eribulin was administered by intraperitoneal injection at $0.1 \mathrm{mg} / \mathrm{kg}$. Saline solution $(0.9 \%)$ was used as the vehicle. After vehicle-treated tumors reached $14 \mathrm{~mm}$ in diameter, the experiment was terminated, and tumors were excised and processed for western blotting analysis (snap-frozen) or fixation in formalin for histological examination (FFPE).

For both cell line and patient-derived xenografts, tumor volume was calculated using the formula: $\mathrm{V}=\left(\pi \times\right.$ length $\times$ width $\left.^{2} / 6\right)$, where length is the longest tumor diameter, and width is the perpendicular diameter. For data analysis, tumor volume was normalized to the volume at which treatment was started and expressed as the fold change relative to this. Statistical analysis was performed using Prism.

\section{Histological analysis}

Histological analysis was performed on $3-\mu \mathrm{m}$ thick tissue sections stained with a standard hematoxylin (Hematoxylin Solution, Gill No.III, Sigma) and eosin (H\&E) staining protocol. All histological slides were digitized at magnification $\times 20(0.46 \mu \mathrm{m} /$ pixel $)$ using a Hamamatsu Nanozoomer 2.0 HT (Hamamatsu Photonics, Hamamatsu, Japan). Digital images in ndp format were submitted for quantitative image analysis using HistoQuest 4.2 (Tissugnostic, Vienna, Austria) software. The HistoQuest nucleus detection algorithm for hematoxylin staining was used as a master channel to identify all tumor cell nuclei and enable cell quantification. The surface area of the tumor was also measured in the system, and the value for tumor cell density was calculated as the number of cells per $\mathrm{mm}^{2}$.

To assess p27 staining, HistoQuest software was optimized to detect individual tumor cells and assess the intensity of DAB staining in the cell nucleus. The software automatically detects tissue that is subjected to color separation to differentiate between blue and brown staining. A nucleus-detection algorithm on the hematoxylin staining was used to detect tumor cell nuclei and was defined as a master channel. A nuclear mask was used to analyze the staining intensity of the chromogen (DAB) in a nonmaster channel. The staining intensity was measured as the mean intensity of all pixels within a cell, with a range of values per pixel from 0 to 255 . 


\section{Statistical analysis}

Gene expression and copy number statistical analyses were performed in the R environment, using several CRAN packages (http://cran.r-project.org/) as described above. Unpaired twosided $t$-tests and one- way ANOVA with Tukey's multiple-comparisons tests were performed using GraphPad Prism software for analysis of the data obtained in all in vitro experiments and in vivo animal studies. For in vitro studies, no samples were processed and then excluded; all completed experiments are reported. For in vivo studies, we estimated that we would need at least six samples per treatment group to see an effect, for a power of $80 \%$ and for the probability of type I error $(\alpha)=0.05$. Experiments were repeated at least twice to confirm treatment response. The sample size for all in vitro experiments was not chosen with consideration of the power needed to detect a prespecified effect size. For in vivo studies, the total number of mice per group is indicated. Mice were excluded from the study if body weight was reduced during treatment by more than $15 \%$ as compared to that at the start of treatment. For mouse studies, investigators were blinded to the group allocation during the experiment and drug treatment. Investigators were also blinded when assessing the outcome by immunohistochemistry. Mice were randomized to treatment groups when tumors reached a predetermined diameter on a per experiment basis, as described above. For each data set, the data meet the assumptions of the statistical test used, as determined by distribution and variance.

\section{Supplementary Material}

Refer to Web version on PubMed Central for supplementary material.

\section{Acknowledgments}

This research was supported by the Breast Cancer Now funding (A.N.T.) at King's College London and the Institute of Cancer Research London, the National Institute for Health Research Biomedical Research Centre based at Guy's and St Thomas' NHS Foundation Trust and King's College London, the US National Institutes of Health grant P30 CA008748 (M.S.) and the Breast Cancer Research Foundation (M.S.). The PDX studies were supported by a 'GHD-pink' research support via the FERO Foundation (V.S.). Patient samples and data were provided by King's Health Partners Cancer Biobank, London, UK, which is supported by the Experimental Cancer Medicine Centre at King's College London and the Department of Health via the National Institute for Health Research comprehensive Biomedical Research Centre award. We thank S. Swift, S. Utting and M. Ferrao for technical and administrative assistance, AstraZeneca for providing AZD1208, D. Huszar (AstraZeneca) for providing information on the use of AZD1208, H. Mirza (King's College London) for his support with the bioinformatics analysis, J. Hurst (The Institute of Cancer Research London) for helping with the NanoString nCounter PanCancer Pathway analysis, M. Dowsett (Institute of Cancer Research and Royal Marsden Hospital National Institute for Health Research comprehensive Biomedical Research Centre London) for providing staff and infrastructure support for the NanoString NCounter work, T. Tenev (Institute of Cancer Research) for providing MDA-MB-231 containing the BCL2 pTIPZ overexpression vector and the Guy's Hospital Pharmacy for providing the chemotherapeutic drugs.

\section{References}

1. Reis-Filho JS, Tutt AN. Triple-negative tumors: a critical review. Histopathology. 2008; 52:108118. [PubMed: 18171422]

2. Symmans WF, et al. Measurement of residual breast cancer burden to predict survival after neoadjuvant chemotherapy. J Clin Oncol. 2007; 25:4414-4422. [PubMed: 17785706]

3. Masuda H, et al. Differential response to neoadjuvant chemotherapy among seven triple-negative breast cancer molecular subtypes. Clin Cancer Res. 2013; 19:5533-5540. [PubMed: 23948975] 
4. Balko JM, et al. Molecular profiling of the residual disease of triple-negative breast cancers after neoadjuvant chemotherapy identifies actionable therapeutic targets. Cancer Discov. 2014; 4:232245. [PubMed: 24356096]

5. Labisso WL, et al. MYC directs transcription of MCL1 and EIF4E genes to control sensitivity of gastric cancer cells toward HDAC inhibitors. Cell Cycle. 2012; 11:1593-1602. [PubMed: 22456335]

6. Andre F, et al. Molecular characterization of breast cancer with high-resolution oligonucleotide comparative genomic hybridization array. Clin Cancer Res. 2009; 15:441-451. [PubMed: 19147748]

7. Bergamaschi A, et al. Distinct patterns of DNA copy-number alteration are associated with different clinicopathological features and gene expression subtypes of breast cancer. Genes Chromosom Cancer. 2006; 45:1033-1040. [PubMed: 16897746]

8. Turner $\mathrm{N}$, et al. Integrative molecular profiling of triple-negative breast cancers identifies amplicon drivers and potential therapeutic targets. Oncogene. 2010; 29:2013-2023. [PubMed: 20101236]

9. Nawijn MC, Alendar A, Berns A. For better or for worse: the role of Pim oncogenes in tumorigenesis. Nat Rev Cancer. 2011; 11:23-34. [PubMed: 21150935]

10. Wang J, et al. Pim1 kinase synergizes with c-MYC to induce advanced prostate carcinoma. Oncogene. 2010; 29:2477-2487. [PubMed: 20140016]

11. Keeton EK, et al. AZD1208, a potent and selective pan-Pim kinase inhibitor, demonstrates efficacy in preclinical models of acute myeloid leukemia. Blood. 2014; 123:905-913. [PubMed: 24363397]

12. Laird PW, et al. In vivo analysis of Pim1 deficiency. Nucleic Acids Res. 1993; 21:4750-4755. [PubMed: 8233823]

13. Magnuson NS, Wang Z, Ding G, Reeves R. Why target PIM1 for cancer diagnosis and treatment? Future Oncol. 2010; 6:1461-1478. [PubMed: 20919829]

14. Narlik-Grassow M, Blanco-Aparicio C, Carnero A. The PIM family of serine-threonine kinases in cancer. Med Res Rev. 2014; 34:136-159. [PubMed: 23576269]

15. Aceto N, et al. Tyrosine phosphatase SHP2 promotes breast cancer progression and maintains tumor-initiating cells via activation of key transcription factors and a positive-feedback signaling loop. Nat Med. 2012; 18:529-537. [PubMed: 22388088]

16. Speers $\mathrm{C}$, et al. Identification of novel kinase targets for the treatment of estrogen-receptor-negative breast cancer. Clin Cancer Res. 2009; 15:6327-6340. [PubMed: 19808870]

17. Lehmann BD, et al. Identification of human triple-negative breast cancer subtypes and preclinical models for selection of targeted therapies. J Clin Invest. 2011; 121:2750-2767. [PubMed: 21633166]

18. de Rinaldis E, et al. Integrated genomic analysis of triple-negative breast cancers reveals novel microRNAs associated with clinical and molecular phenotypes and sheds light on the pathways they control. BMC Genomics. 2013; 14:643. [PubMed: 24059244]

19. Gazinska P, et al. Comparison of basal-like triple-negative breast cancer defined by morphology, immunohistochemistry and transcriptional profiles. Mod Pathol. 2013; 26:955-966. [PubMed: 23392436]

20. The Cancer Genome Atlas. Comprehensive molecular portraits of human breast tumors. Nature. 2012; 490:61-70. [PubMed: 23000897]

21. Curtis C, et al. The genomic and transcriptomic architecture of 2,000 breast tumors reveals novel subgroups. Nature. 2012; 486:346-352. [PubMed: 22522925]

22. Parker JS, et al. Supervised risk predictor of breast cancer based on intrinsic subtypes. J Clin Oncol. 2009; 27:1160-1167. [PubMed: 19204204]

23. Neve RM, et al. A collection of breast cancer cell lines for the study of functionally distinct cancer subtypes. Cancer Cell. 2006; 10:515-527. [PubMed: 17157791]

24. Canbay E. Erb-B2 homodimerization inhibits $M U C 1$ transcription in cultured human mammary epithelial cells. Cell Biol Int. 2003; 27:477-481. [PubMed: 12798775]

25. Aho TL, et al. Pim1 kinase promotes inactivation of the pro-apoptotic Bad protein by phosphorylating it on the Ser112 gatekeeper site. FEBS Lett. 2004; 571:43-49. [PubMed: 15280015] 
26. Lilly M, Sandholm J, Cooper JJ, Koskinen PJ, Kraft A. The PIM1 serine kinase prolongs survival and inhibits apoptosis-related mitochondrial dysfunction in part through a BCL-2-dependent pathway. Oncogene. 1999; 18:4022-4031. [PubMed: 10435626]

27. Juin P, Geneste O, Gautier F, Depil S, Campone M. Decoding and unlocking the BCL-2 dependency of cancer cells. Nat Rev Cancer. 2013; 13:455-465. [PubMed: 23783119]

28. Montero J, et al. Drug-induced death signaling strategy rapidly predicts cancer response to chemotherapy. Cell. 2015; 160:977-989. [PubMed: 25723171]

29. Verbeek S, et al. Mice bearing the E $\mu-M y c$ and E $\mu$-Pim1 transgenes develop pre-B-cell leukemia prenatally. Mol Cell Biol. 1991; 11:1176-1179. [PubMed: 1990273]

30. Zippo A, De Robertis A, Serafini R, Oliviero S. PIM1-dependent phosphorylation of histone H3 at Ser10 is required for MYC-dependent transcriptional activation and oncogenic transformation. Nat Cell Biol. 2007; 9:932-944. [PubMed: 17643117]

31. Sears R, et al. Multiple Ras-dependent phosphorylation pathways regulate Myc protein stability. Genes Dev. 2000; 14:2501-2514. [PubMed: 11018017]

32. Noguchi K, et al. Regulation of c-Myc through phosphorylation at Ser-62 and Ser-71 by c-Jun Nterminal kinase. J Biol Chem. 1999; 274:32580-32587. [PubMed: 10551811]

33. Wang X, et al. Phosphorylation regulates c-Myc's oncogenic activity in the mammary gland. Cancer Res. 2011; 71:925-936. [PubMed: 21266350]

34. Chandriani S, et al. A core MYC gene expression signature is prominent in basal-like breast cancer but only partially overlaps the core serum response. PLoS One. 2009; 4:e6693. [PubMed: 19690609]

35. Horiuchi D, et al. MYC pathway activation in triple-negative breast cancer is synthetic lethal with CDK inhibition. J Exp Med. 2012; 209:679-696. [PubMed: 22430491]

36. Grigoriadis A, et al. Molecular characterization of cell line models for triple-negative breast cancers. BMC Genomics. 2012; 13:619. [PubMed: 23151021]

37. Morishita D, Katayama R, Sekimizu K, Tsuruo T, Fujita N. Pim kinases promote cell cycle progression by phosphorylating and down-regulating p27Kip1 at the transcriptional and posttranscriptional levels. Cancer Res. 2008; 68:5076-5085. [PubMed: 18593906]

38. Miura K, et al. Involvement of EphA2-mediated tyrosine phosphorylation of Shp2 in Shp2regulated activation of extracellular-signal-regulated kinase. Oncogene. 2013; 32:5292-5301. [PubMed: 23318428]

39. Hudis CA, Gianni L. Triple-negative breast cancer: an unmet medical need. Oncologist. 2011; 16(Suppl 1):1-11.

40. Kirschner AN, et al. PIM kinase inhibitor AZD1208 for treatment of MYC-driven prostate cancer. J Natl Cancer Inst. 2014; 107:dju407. [PubMed: 25505253]

41. Turner NC, Reis-Filho JS. Tackling the diversity of triple-negative breast cancer. Clin Cancer Res. 2013; 19:6380-6388. [PubMed: 24298068]

42. Morton JP, Sansom OJ. MYC-y mice: from tumor initiation to therapeutic targeting of endogenous MYC. Mol Oncol. 2013; 7:248-258. [PubMed: 23523308]

43. Horiuchi D, et al. PIM kinase inhibition presents a novel targeted therapy against triple-negative breast tumors with elevated MYC expression. Nat Med. 2016; 22:XXX-XXX. [CE and Production: This manuscript is being published in the same AOP batch, and this reference will need to be updated with the correct title and publication details.]

44. Petrocca F, et al. A genome-wide siRNA screen identifies proteasome addiction as a vulnerability of basal-like triple-negative breast cancer cells. Cancer Cell. 2013; 24:182-196. [PubMed: 23948298]

45. Garcia PD, et al. Pan-PIM kinase inhibition provides a novel therapy for treating hematologic cancers. Clin Cancer Res. 2014; 20:1834-1845. [PubMed: 24474669]

46. Malinen M, et al. Proto-oncogene PIM1 is a novel estrogen receptor target associating with highgrade breast tumors. Mol Cell Endocrinol. 2013; 365:270-276. [PubMed: 23142699]

47. Raab, MS., et al. Phase 1 study of the novel pan-PIM kinase inhibitor LGH447 in patients with relapsed or refractory multiple myeloma. 55th ASH Annual meeting and exposition; New Orleans, LA: 2013. 
48. McEachern, KA., et al. Preclinical and clinical pharmacodynamics of pan-PIM inhibition by AZD1208 in acute myeloid leukemia: assessment of PIM isoform dependency for BAD and 4EBP1 phosphorylation. 56th ASH Annual meeting and exposition; San Francisco, CA: 2014.

49. Mori S, et al. Utilization of pathway signatures to reveal distinct types of B lymphoma in the E $\mu$ myc model and human diffuse large-B-cell lymphoma. Cancer Res. 2008; 68:8525-8534. [PubMed: 18922927]

50. Bild AH, et al. Oncogenic pathway signatures in human cancers as a guide to targeted therapies. Nature. 2006; 439:353-357. [PubMed: 16273092]

51. Zeller KI, Jegga AG, Aronow BJ, O’Donnell KA, Dang CV. An integrated database of genes responsive to the Myc oncogenic transcription factor: identification of direct genomic targets. Genome Biol. 2003; 4:R69. [PubMed: 14519204]

52. O'Donnell KA, et al. Activation of transferrin receptor 1 by c-Myc enhances cellular proliferation and tumorigenesis. Mol Cell Biol. 2006; 26:2373-2386. [PubMed: 16508012]

53. Fernandez PC, et al. Genomic targets of the human c-Myc protein. Genes Dev. 2003; 17:11151129. [PubMed: 12695333]

54. Kim YH, et al. Combined microarray analysis of small-cell lung cancer reveals altered apoptotic balance and distinct expression signatures of MYC family gene amplification. Oncogene. 2006; 25:130-138. [PubMed: 16116477]

55. Lastowska M, et al. Comprehensive genetic and histopathologic study reveals three types of neuroblastoma tumors. J Clin Oncol. 2001; 19:3080-3090. [PubMed: 11408505]

56. Lee JS, et al. Application of comparative functional genomics to identify best-fit mouse models to study human cancer. Nat Genet. 2004; 36:1306-1311. [PubMed: 15565109]

57. Menssen A, Hermeking H. Characterization of the c-MYC-regulated transcriptome by SAGE: identification and analysis of c-MYC target genes. Proc Natl Acad Sci USA. 2002; 99:6274-6279. [PubMed: 11983916]

58. Schlosser I, et al. Dissection of transcriptional programs in response to serum and c-Myc in a human B cell line. Oncogene. 2005; 24:520-524. [PubMed: 15516975]

59. Schuhmacher M, et al. The transcriptional program of a human B cell line in response to Myc. Nucleic Acids Res. 2001; 29:397-406. [PubMed: 11139609]

60. Yu D, Cozma D, Park A. Thomas-Tikhonenko A: Functional validation of genes implicated in lymphomagenesis: an in vivo selection assay using a Myc-induced B cell tumor. Ann NY Acad Sci. 2005; 1059:145-159. [PubMed: 16382050]

61. Hammond ME, et al. American Society of Clinical Oncology-College Of American Pathologists guideline recommendations for immunohistochemical testing of estrogen and progesterone receptors in breast cancer. J Clin Oncol. 2010; 28:2784-2795. [PubMed: 20404251]

62. Van Loo P, et al. Allele-specific copy-number analysis of tumors. Proc Natl Acad Sci USA. 2010; 107:16910-16915. [PubMed: 20837533]

63. Rasmussen $\mathrm{M}$, et al. Allele-specific copy-number analysis of tumor samples with aneuploidy and tumor heterogeneity. Genome Biol. 2011; 12:R108. [PubMed: 22023820]

64. Meerbrey KL, et al. The pINDUCER lentiviral toolkit for inducible RNA interference in vitro and in vivo. Proc Natl Acad Sci USA. 2011; 108:3665-3670. [PubMed: 21307310]

65. Franken NA, Rodermond HM, Stap J, Haveman J, van Bree C. Clonogenic assay of cells in vitro. Nat Protoc. 2006; 1:2315-2319. [PubMed: 17406473] 


\section{Editorial summary}

AOP: In triple-negative breast cancer, the kinase PIM1, which is highly expressed, functions through the transcription factor c-MYC to promote tumor cell survival and growth. 
a

Guy's Hospital TNBC Enriched Cohort

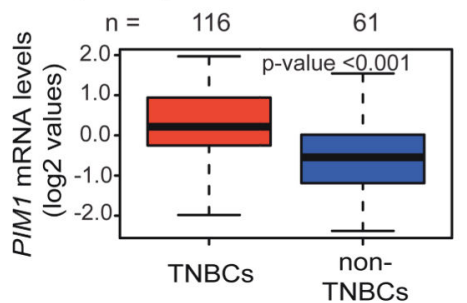

b

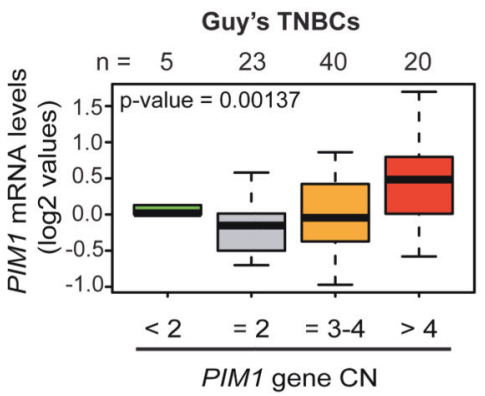

C

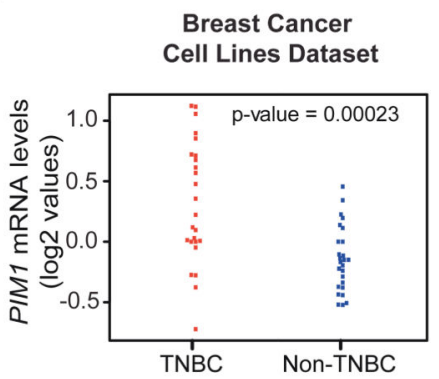

d
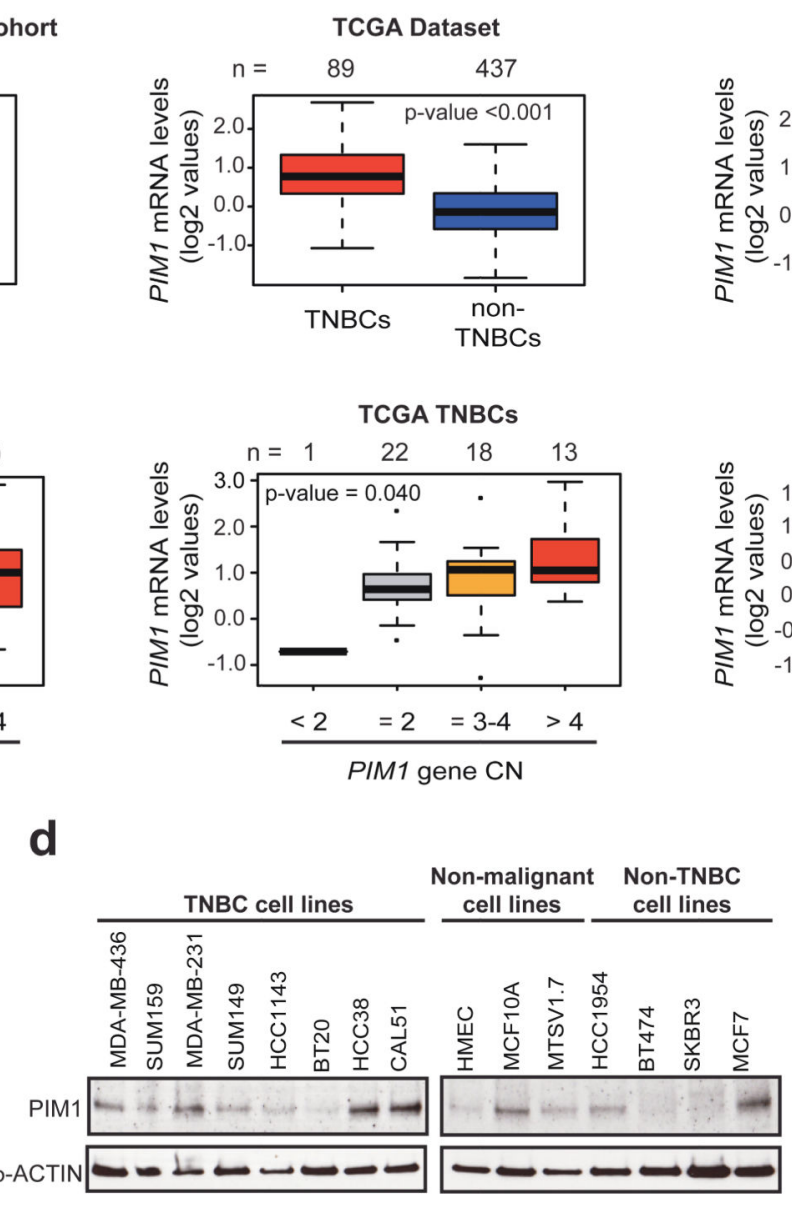
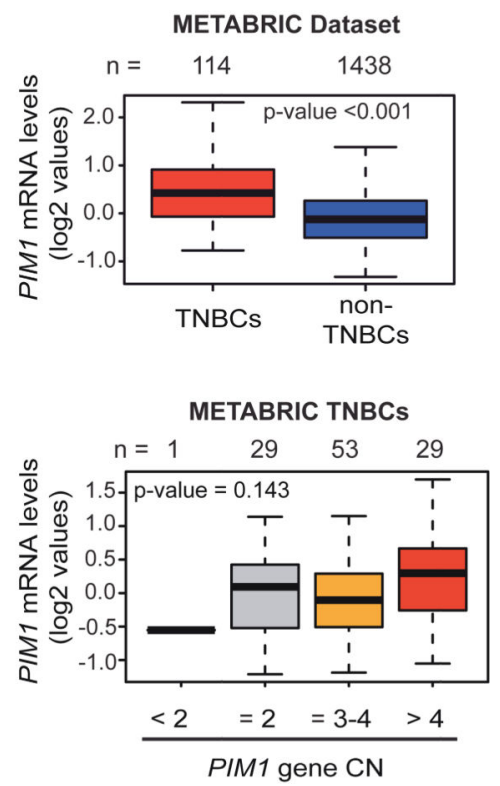

e

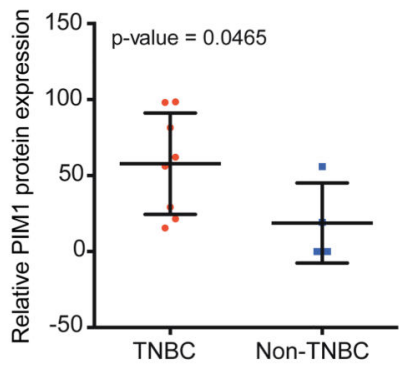

Figure 1. PIM1 gene expression is upregulated in TNBC and associated with increased PIM1 gene copy number.

(a) PIM1 gene expression in the Guy's Hospital TNBC-enriched cohort18,19 (left), TCGA

Breast20 (middle) and METABRIC21 (right) data sets. The cohorts were divided into

TNBCs (red) and non-TNBCs (blue) according to their immunohistochemistry (IHC)-

defined receptor status. Gene expression is reported as median-centered expression $\log _{2}$

values. The number of patients $(n)$ per group is indicated. $P$ values were determined using a

Wilcoxon rank-sum test. (b) Absolute PIM1 gene copy number (CN) plotted against PIM1 gene expression values in TNBCs from the Guy's Hospital (left), TCGA (middle) and METABRIC (right) data sets. Tumors were segregated according to their PIM1 gene CN status: high amplification $(\mathrm{CN}>4)$, moderate gain $(\mathrm{CN}=3$ or 4$)$, neutral copy number $(\mathrm{CN}$ $=2)$ or deletion $(\mathrm{CN}<2)$. The number of samples $(n)$ per group is indicated. $P$ values were determined using the Kruskal-Wallis analysis of variance (ANOVA) test. (c) PIM1 gene expression in the Neve et al. cancer cell line expression data set23. Cell lines were divided into TNBCs (red) and non-TNBCs (blue) according to their receptor status. $P=0.00023$ by Welch's $t$-test (Satterthwaite's approximation). (d) PIM1 protein expression, as assessed by western blotting, in a panel of breast cancer cell lines and cell lines derived from nonmalignant breast epithelial tissue (hereafter referred to as nonmalignant cell lines). (e) 
Relative PIM1 protein expression in TNBC versus non-TNBC cell lines, as quantified by densitometry from three independent experiments. $\beta$-actin was used a loading control for normalization. $P=0.0465$ by two-tailed unpaired $t$-test. 
a

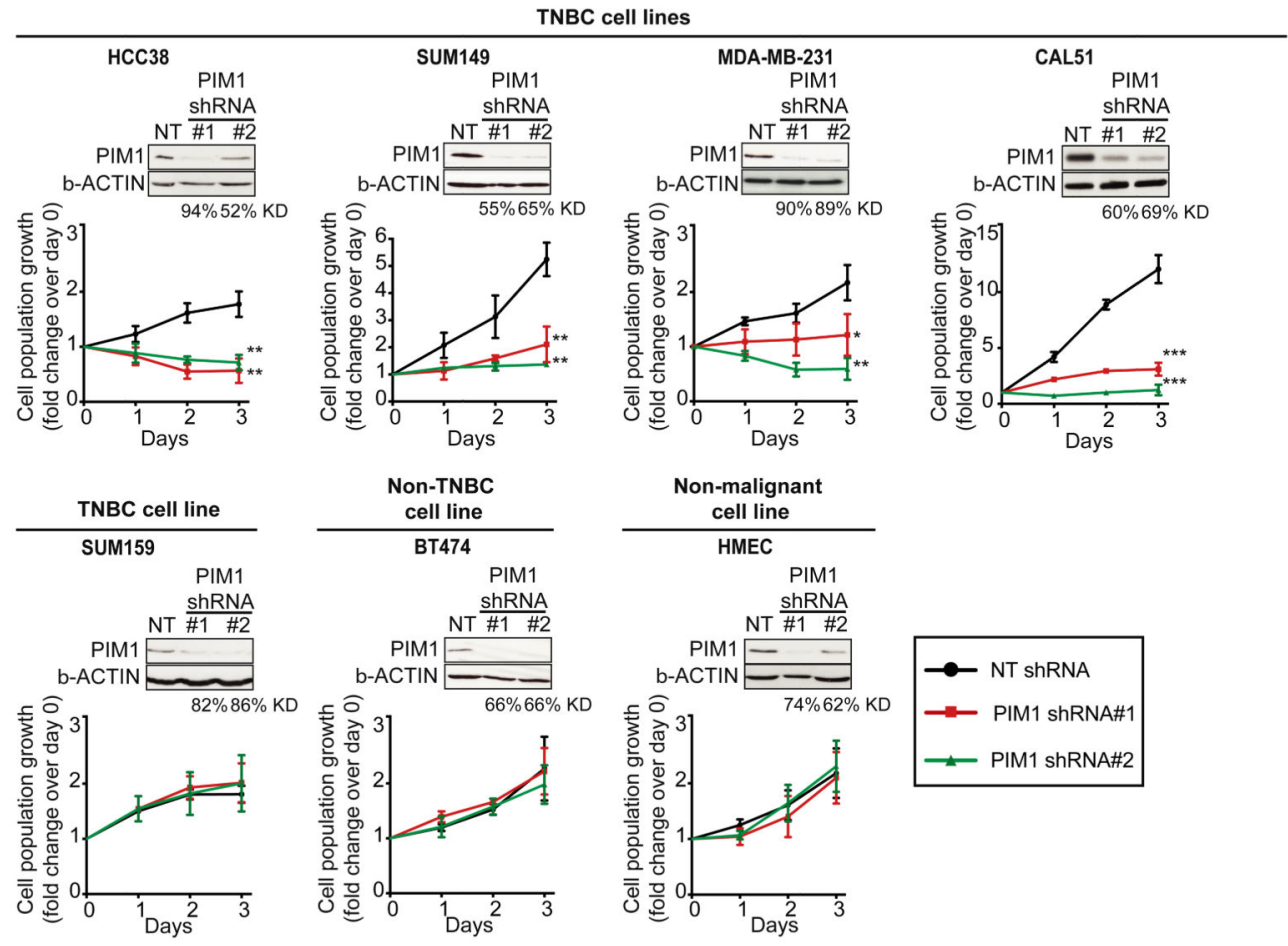

b
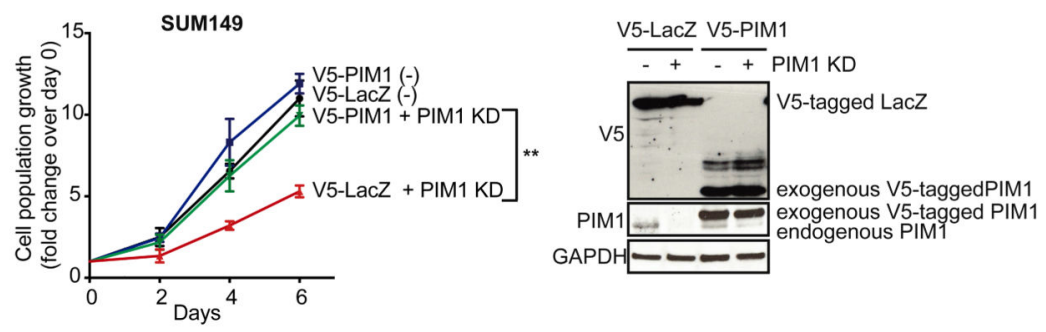

C
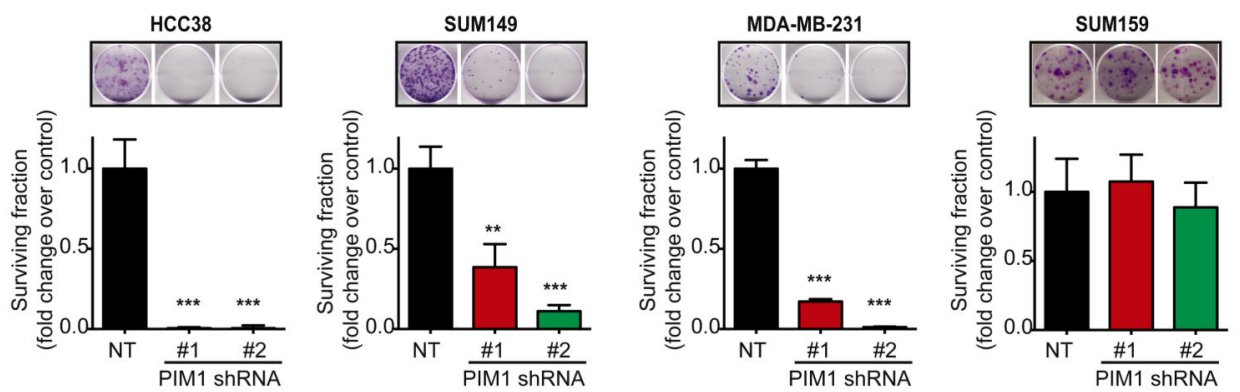

Figure 2. PIM1 supports cell population growth and clonogenic survival of TNBC cells.

(a) PIM1 expression, as determined by western blotting, (top) and quantification of cell population growth over time (bottom) of the indicated TNBC (HCC38, SUM149, MDAMB-231, CAL51 and SUM159), non-TNBC (BT474) and nonmalignant (HMEC) cell lines (bottom) after treatment with two distinct PIM1-specific shRNAs (shPIM1\#1 and shPIM1\#2) or a nontargeting (shNT) control shRNA. In the western blot analyses, the percentage of PIM1 knockdown (KD) was quantified by densitometry. $\beta$-actin protein was used as a loading control. (b) Left, cell population growth of SUM149 cells that were 
infected with lentivirus stably overexpressing shPIM1\#1-resistant V5-tagged PIM1 (V5PIM1 ${ }^{\mathrm{R}}$ ) or control V5-tagged LacZ (V5-LacZ), and then superinfected with the doxycycline-inducible shPIM1\#1 construct. Endogenous PIM1 knockdown was induced with doxycycline (+PIM1 KD). Cells not exposed to doxycycline were used as a control (-). Right, expression of V5-tagged PIM1 and LacZ proteins, and knockdown of endogenous PIM1, were confirmed by western blotting. GAPDH was used as a loading control. (c) Clonogenic survival of HCC38, SUM149, MDA-MB-231 and SUM159 cells after treatment with PIM1- specific shRNAs or with shNT. Top, representative images of colony-formation assays. Scale bars, $10 \mathrm{~mm}$. Bottom, surviving fraction values, as determined by the number of colonies for each condition relative to those after expression of shNT. Data in a-c represent the mean \pm s.e.m. values of three independent experiments. The last time points (day 3 in $\mathbf{a}$ and day 6 in $\mathbf{b}$ ) were analyzed by using a two-tailed unpaired $t$-test. $* P<0.05$, $* * P<0.01, * * * P<0.001$. 
a

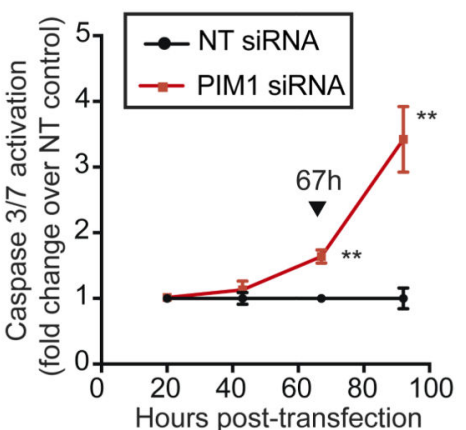

d
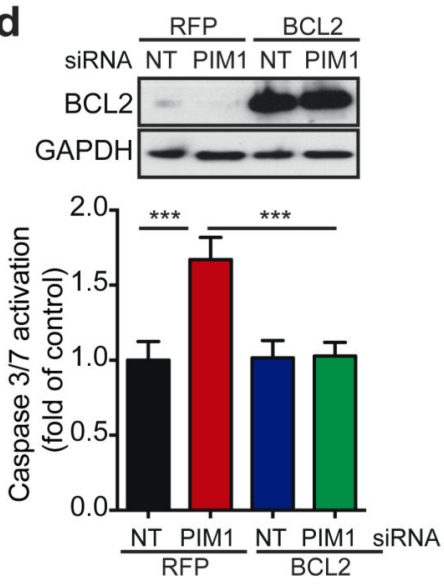

b

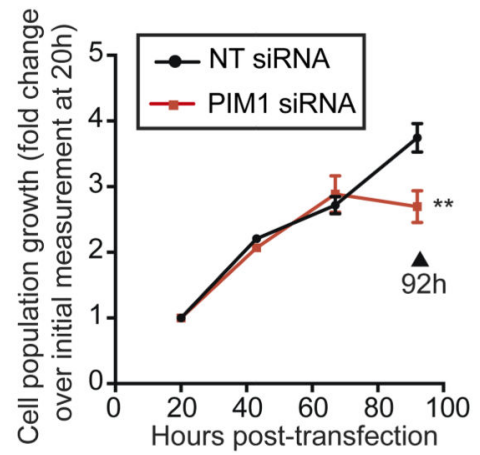

C

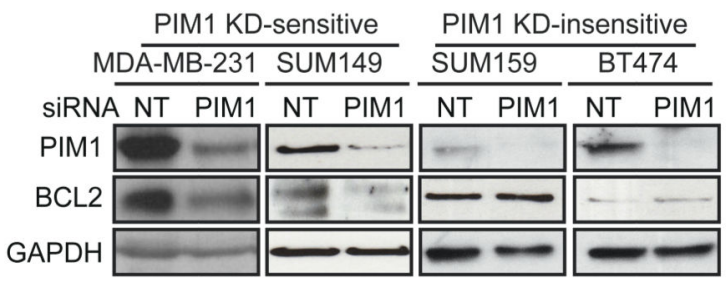

e

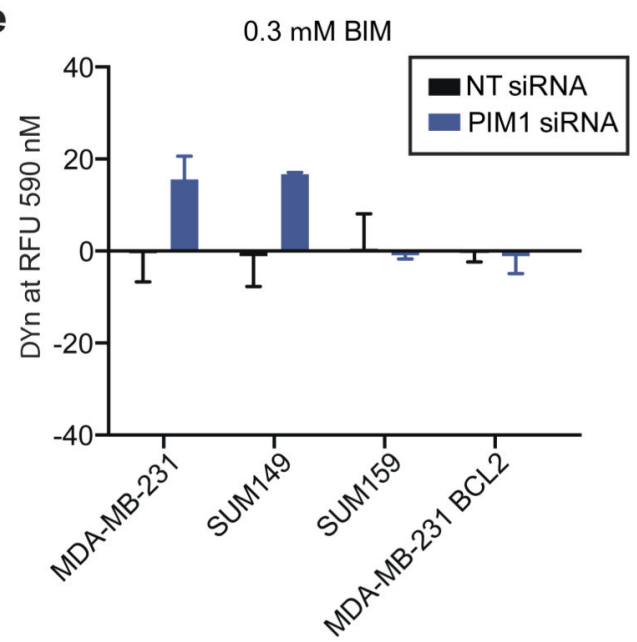

f
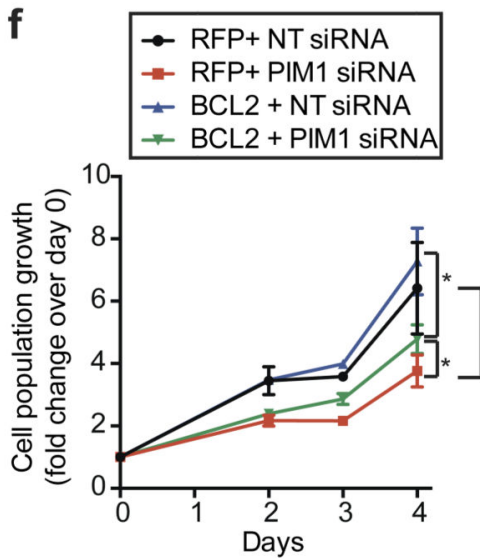

Figure 3. PIM1 inhibits activation of mitochondrial-mediated apoptosis in TNBC cells.

$(\mathbf{a}, \mathbf{b})$ Time course of caspase 3/7 activation (a) and cell population growth (b) of MDAMB-231 cells that were transfected with $20 \mathrm{nM}$ of a PIM1-specific or control (NT) siRNA.

Each data point represents the mean \pm s.d. of three independent biological replicates. $* * P<$ 0.01 by two-tailed unpaired $t$-test. (c) Representative western blot analysis (of $n=3$ ) for PIM1 and BCL2 expression in PIM1-knockdown-sensitive (MDA-MB-231 and SUM149) and PIM1-knockdown-insensitive (SUM159 and BT474) cells after exposure to a PIM1specific or NT siRNA for $4 \mathrm{~d}$. GAPDH expression was used as a loading control. (d) Representative western blot for BCL2 expression (top) and quantification of caspase-3/7 activation (bottom) in MDA-MB-231 cells overexpressing BCL2 or a red fluorescent protein (RFP) control and transfected with a PIM1-specific or NT control siRNA $72 \mathrm{~h}$ after transfection. The data represent the mean \pm s.e.m. values of three independent experiments. $* * * P<0.001$ by one-way ANOVA with Tukey's post hoc test. For the western blotting analysis, GAPDH was used as a loading control. (e) BIM-induced mitochondrial membrane permeabilization $\left(\Delta \Psi_{\mathrm{n}}\right.$ priming, as determined by relative fluorescence units (RFU) at 590 $\mathrm{nm}$ ) achieved with $0.3 \mu \mathrm{M}$ BIM peptide treatment of MDA-MB-231, SUM149, SUM159 or BCL2-overexpressing MDA-MB-231 cells treated with the PIM1- specific or NT siRNA. The data represent the mean \pm s.e.m. values of three independent experiments. (f) Cell population growth of MDA-MB-231 cells overexpressing BCL2 or RFP that were treated 
with the PIM1-specific or NT siRNA. Growth was measured every $2 \mathrm{~d}$, starting at $2 \mathrm{~d}$ after transfection (referred to as day 0 ). The data represent the mean \pm s.e.m. values of three independent experiments. $* P<0.05$ by Welch's ANOVA. 
a

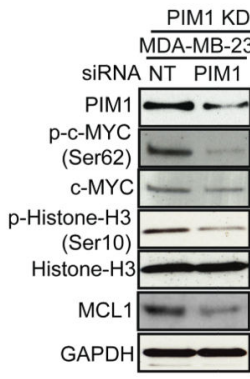

C

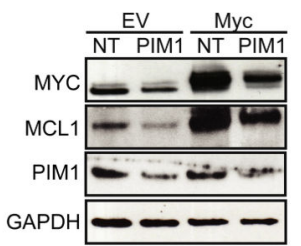

d

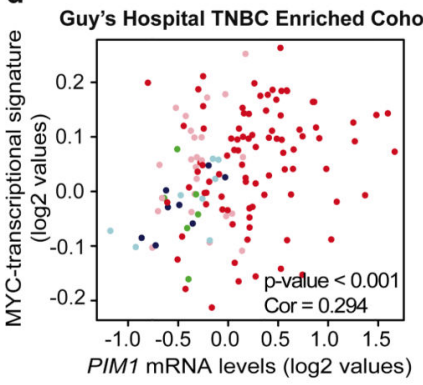

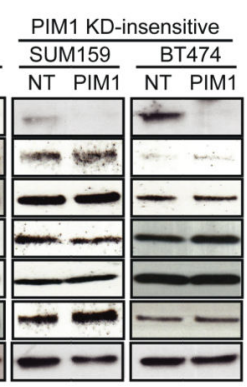

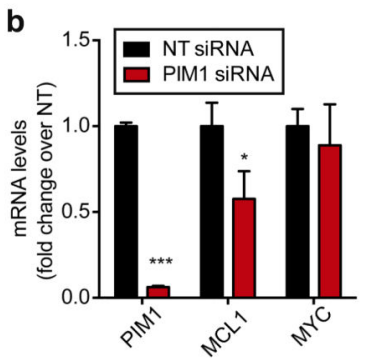

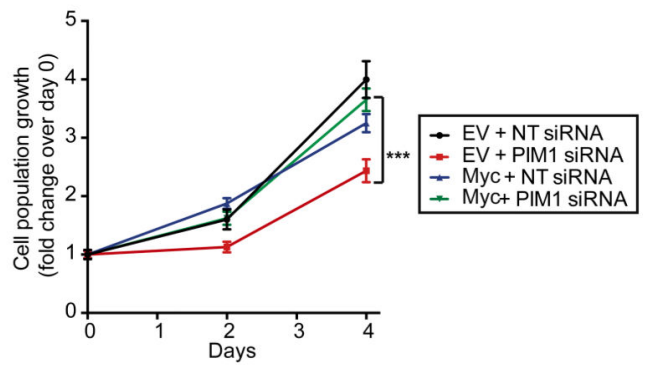
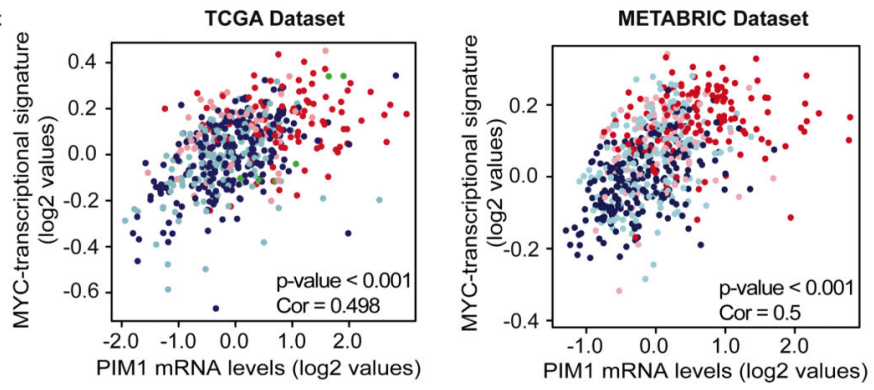

$\bullet$ Basal-like

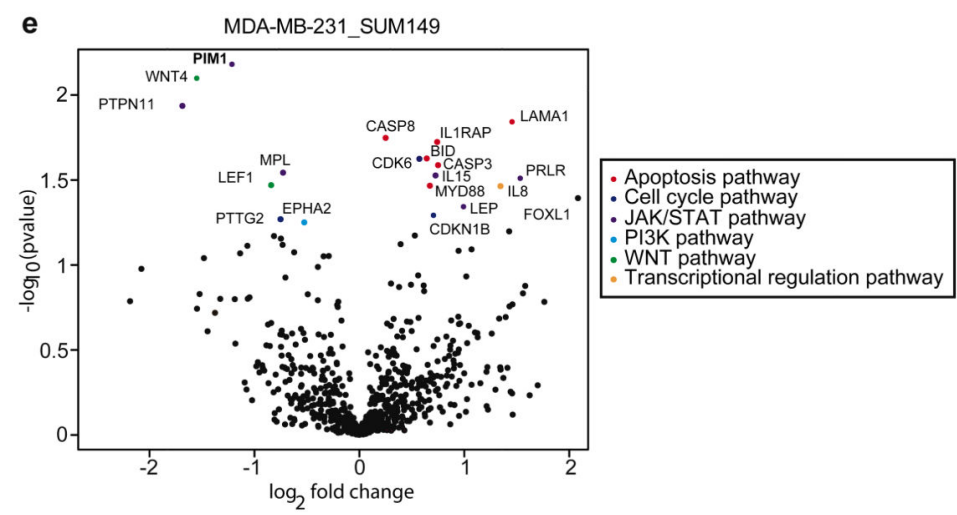

f

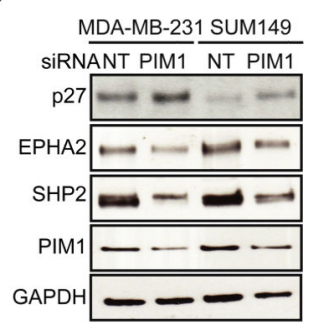

Figure 4. PIM1 functions through the MYC activation pathway.

(a) Western blot analysis (of $n=3$ ) for expression of PIM1, c-MYC, histone H3 and MCL1, and for levels of c-MYC phosphorylation (p-) at Ser62 and histone H3 phosphorylation at Ser10, in PIM1-knockdown-sensitive (MDA-MB-231 and SUM149) and PIM1-knockdowninsensitive (SUM159 and BT474) cells after siRNA-mediated PIM1 knockdown. (b) mRNA levels of PIM1, MCL1 and MYC in MDA-MB-231 cells $72 \mathrm{~h}$ after transfection with a PIM1-specific siRNA, as assessed by qRT-PCR. Expression of the housekeeping gene $G A P D H$ was used for normalization. The data represent the mean \pm s.e.m. values of three 
independent experiments. $* P<0.05$; $* * * P<0.001$; by two- tailed unpaired $t$-test. (c) Representative western blot analysis (of $n=3$ ) (left) and quantification of cell population growth over time (right) in SUM149 doxycycline-induced $M Y C$-overexpressing or emptyvector (EV)-containing control cells, after treatment with a PIM1-specific or NT siRNA. The data represent the mean \pm s.e.m. values of three independent experiments. $* * * P<0.001$ by one-way ANOVA with Tukey's post hoc procedure. (d) PIM1 gene expression is plotted against the Chandriani et al. MYC transcriptional signature34 for the Guy's Hospital (left), TCGA Breast (middle) and METABRIC (right) data sets. Tumor classification by PAM50 molecular intrinsic subtypes is shown. Correlation values and $P$ values were determined using Pearson's correlation. (e) Volcano plot illustrating changes in mRNA expression ( $\log _{2}$ values) for 730 genes in MDA-MB-231 and SUM149 cells that were treated with RNAi (siRNA or shRNA) targeting PIM1 versus cells treated with NT siRNA or shRNA controls, as determined by nCounter PanCancer Pathways gene expression analysis. The top 20 gene expression changes are colored according to their associated signaling pathways, as described by the nCounter PanCancer Pathways code set. (f) Representative western blot analysis (of $n=3$ ) for PIM1, p27, SHP2 and EPHA2 expression in SUM149 and MDAMB-231 cells after treatment with a PIM1-specific or NT siRNA. Throughout, for the western blot analyses GAPDH was used as a loading control. 
a

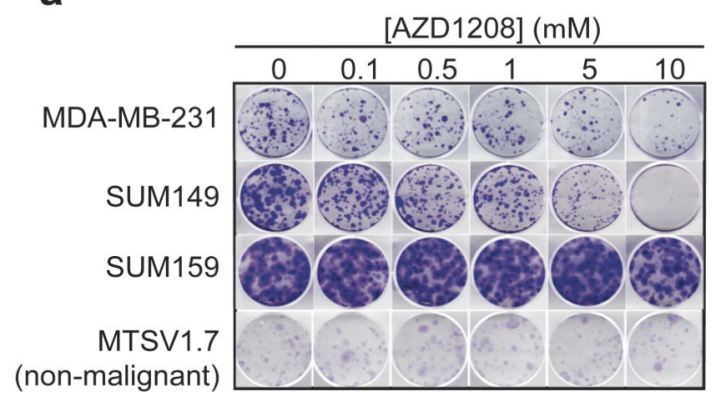

d

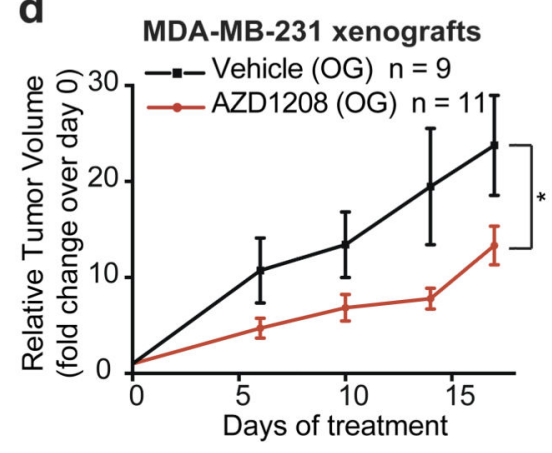

f

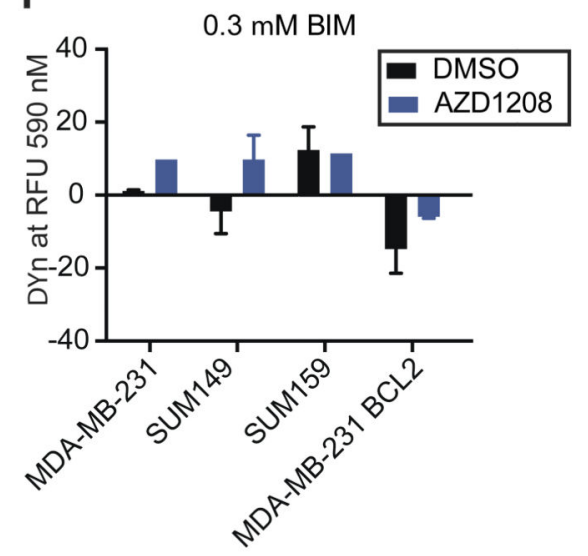

b

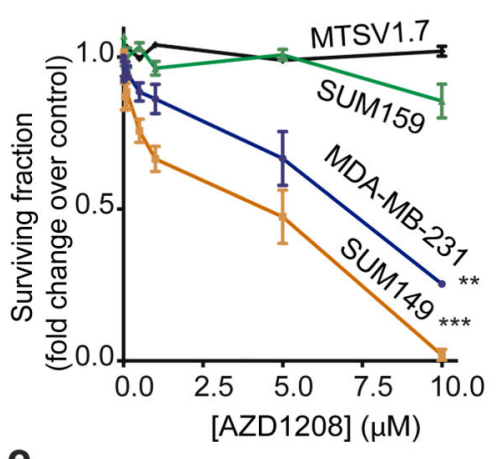

e

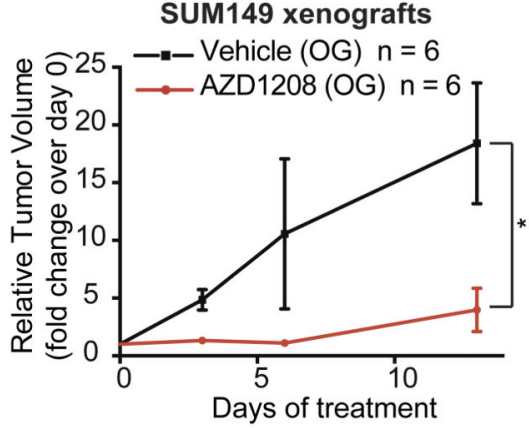

C

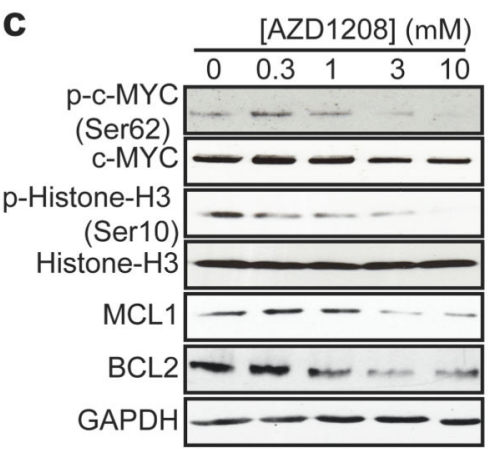

g
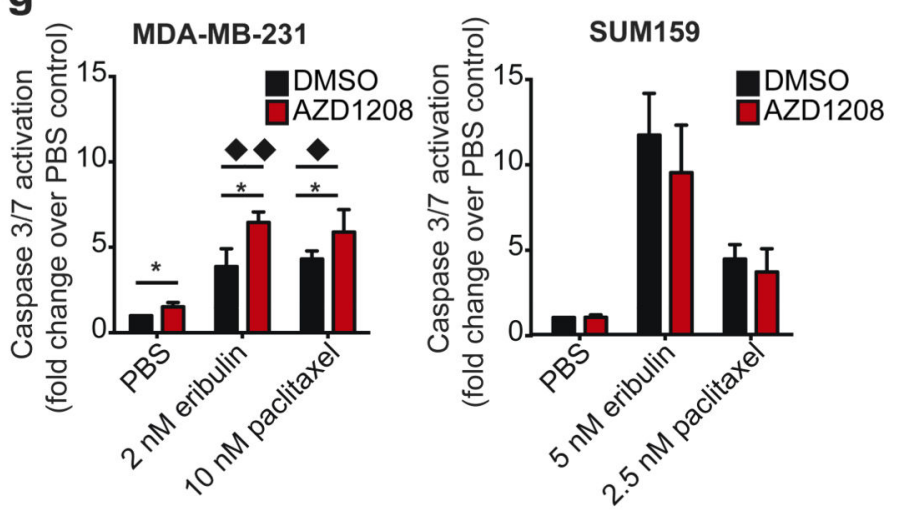

Figure 5. The pan-PIM kinase inhibitor AZD1208 impairs clonogenic survival and reduces tumor growth in in vivo xenograft models of TNBC.

(a,b) Clonogenic survival and growth of MDA-MB-231, SUM159, SUM149 and MTSV1.7 cells after treatment with increasing concentrations of AZD1208, as assessed by colonyformation assays (a), and quantification of the surviving fraction values for each condition, determined from the number of colonies for each condition relative to those in the no-drug control (b). In b, the data represent the mean \pm s.e.m. values of three independent experiments. $* * P<0.01$; $* * * P<0.001$ by linear regression of $\log _{10}$ (AZD1208 concentration) dose response and percentage of survival fraction. Scale bar, $10 \mathrm{~mm}$. (c) Western blot analysis (of $n=3$ ) for levels of c-MYC phosphorylation at Ser62, total c-MYC, histone H3 phosphorylation at Ser10, total histone H3, MCL1 and BCL2 in SUM149 cells following $48 \mathrm{~h}$ of incubation with increasing concentrations of AZD1208. GAPDH was used 
as a loading control. (d,e) Relative tumor volume over time in MDA-MB-231 (d) and SUM149 (e) tumor-bearing mice following treatment with AZD1208 or vehicle control by oral gavage $(\mathrm{OG})$. Relative tumor volume was calculated by normalizing tumor volumes at the indicated time points to those at the start of the treatment (day 0 ). The data represent the mean \pm s.e.m. The number of treated mice $(n)$ per group is shown. The last time points were analyzed by using a two- tailed unpaired $t$-test. $* P<0.05$. (f) BIM-induced mitochondrial membrane permeabilization in the presence of $0.3 \mu \mathrm{M}$ BIM peptide in MDA-MB-231, SUM149, SUM159 and BCL2-overexpressing MDA-MB-231 cells that were treated with 1 $\mu \mathrm{M}$ AZD1208 or DMSO control. The data represent the mean \pm s.e.m. values of three independent experiments. (g) Caspase 3/7 activation levels in MDA-MB-231 (left) and SUM159 (right) cells following $72 \mathrm{~h}$ of incubation with eribulin or paclitaxel, in the presence or absence of $3 \mu \mathrm{M}$ AZD1208. The data represent the mean \pm s.e.m. values of three independent experiments. $* P<0.05$ by two-tailed unpaired $t$-test, and $\diamond \diamond P<0.01$ and $\diamond P<$ 0.05 by one-way ANOVA with Tukey's post hoc procedure. 

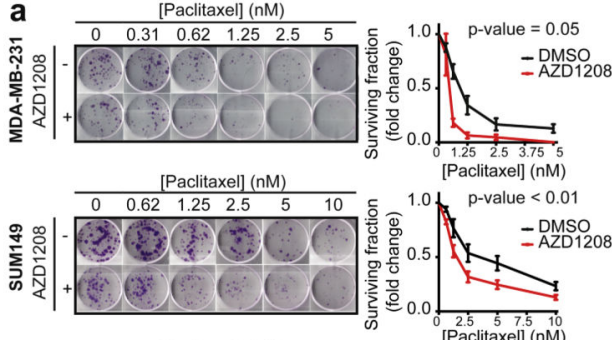

[Paclitaxel] (nM)
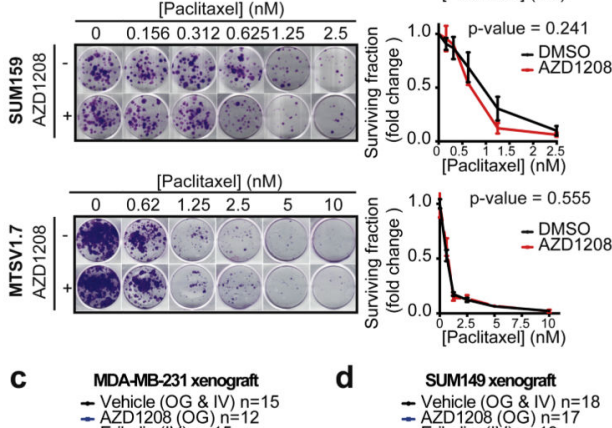

(OG \& IV) $n=15$
$(O G) n=12$
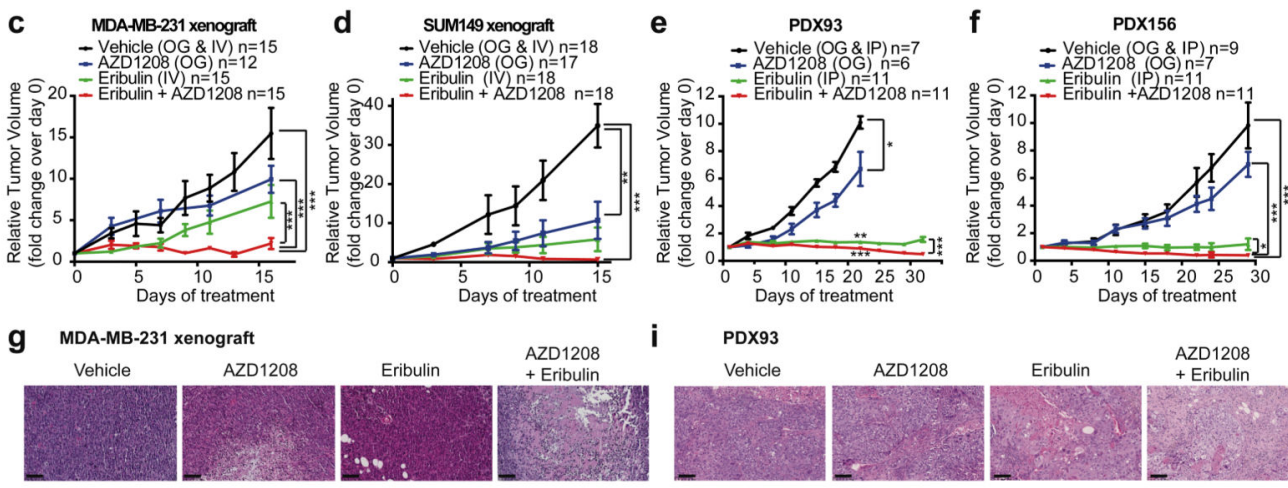

h sum149 xenograft Vehicle

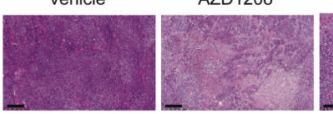

k
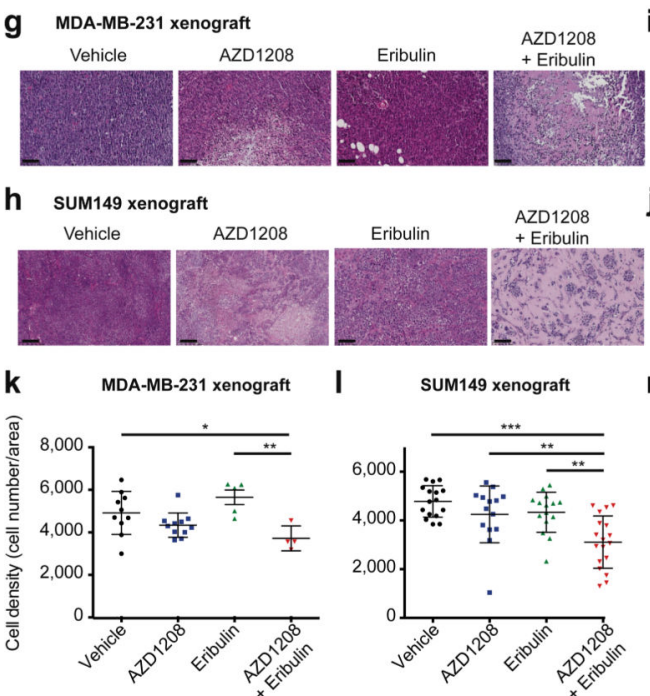

i $\quad$ PDX93
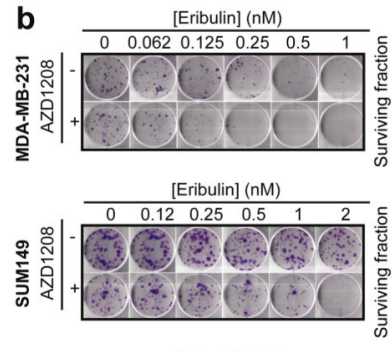

[Eribulin] (nM)

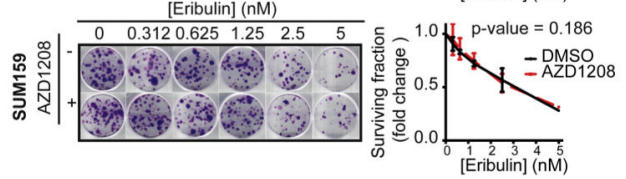

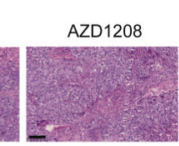

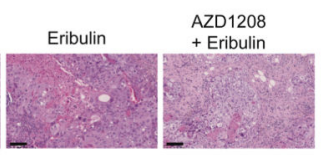

j PDX156

Vehicle
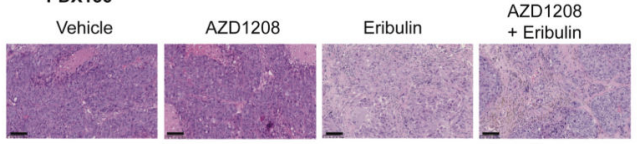

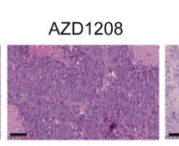

m
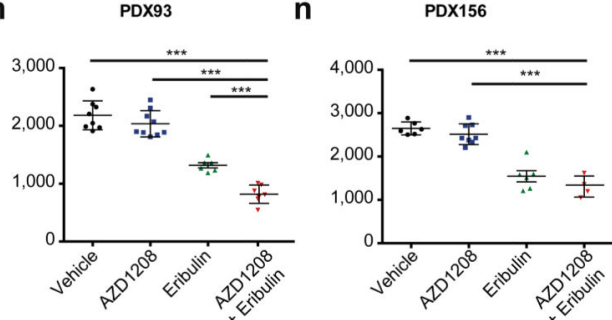

Figure 6. The pan-PIM kinase inhibitor AZD1208 enhances responses to chemotherapy in TNBC cells and xenografts.

(a,b) Representative images of colony-formation assays (left) and quantification of surviving fraction values (relative to the number of colonies in the DMSO control) (right) for the indicated cell lines after treatment with increasing doses of paclitaxel (a) or eribulin (b) in the presence of AZD1208 ( $3 \mu \mathrm{M}$ for MDA-MB-231, SUM159 and MTSV1.7 cells, and 1 $\mu \mathrm{M}$ for SUM149 cells) (+) or DMSO (-). The data represent the mean \pm s.e.m. values of the surviving fraction for each chemotherapy dose point with DMSO control or AZD1208 
across at least three independent experiments. $P$ values were determined by the paired $t$-test. Scale bars, $10 \mathrm{~mm}$. (c,d) Relative tumor volume over time in mice bearing MDA-MB-231 (c) or SUM149 (d) xenografts following treatment with AZD1208 by oral gavage (OG), eribulin by intravenous tail-vein injection (IV), or a combination of eribulin and AZD1208. (e,f) Relative tumor volume over time in mice bearing patient-derived xenograft tumors PDX93 (e) or PDX156 (f) following treatment of host mice with AZD1208 by oral gavage, eribulin by intraperitoneal injection (IP), or a combination of eribulin and AZD1208. Vehicle treatment was used as a control. In $\mathbf{c}-\mathbf{f}$, the number of treated mice $(n)$ per group is indicated. Data represent the mean \pm s.e.m. $* P<0.05$; $* * P<0.01$; *** $P<0.001$; by Welch's ANOVA. (g-j) Representative H\&E-stained images of MDA-MB-231 xenografts (vehicle-treated, $n=10$; AZD1208-treated, $n=11$; eribulin-treated, $n=5$; combinationtreated, $n=4)(\mathrm{g})$, SUM149 xenografts (vehicle-treated, $n=16$; AZD1208-treated, $n=14$; eribulin-treated, $n=15$; combination-treated, $n=18)(\mathbf{h})$, PDX93 tumors (vehicle-treated, $n$ = 8; AZD1208-treated, $n=9$; eribulin-treated, $n=6$; combination-treated, $n=7)$ (i) and PDX156 tumors (vehicle-treated, $\mathrm{n}=6$; AZD1208-treated, $n=8$; eribulin-treated, $n=6$; combination-treated, $n=4)(\mathbf{j})$ at the end of the indicated treatments. Scale bars, $100 \mu \mathrm{m}$. $(\mathbf{k}-\mathbf{n})$ Quantification of cell density (number of tumor cells/area) of MDA-MB-231 xenografts (k), SUM149 xenografts (I), PDX93 tumors (m) and PDX156 tumors (n) at the end of the indicated treatments. Each symbol represents an individual tumor. Data in $\mathbf{k}-\mathbf{n}$ were analyzed by one-way ANOVA with Tukey's multiple-comparisons test. $* P<0.05, * * P$ $<0.01, * * * P<0.001$. 